\title{
Nature and Potential Impact of Alcohol Health Warning Labels: A Scoping Review
}

\author{
Daša Kokole ${ }^{1, *(D)}$, Peter Anderson ${ }^{1,2}$ and Eva Jané-Llopis 1,3,4 \\ 1 Department of Health Promotion, CAPHRI Care and Public Health Research Institute, Maastricht University, \\ POB 616, 6200 MD Maastricht, The Netherlands; peteranderson.mail@gmail.com (P.A.); \\ eva.jane@esade.edu (E.J.-L.) \\ 2 Population Health Sciences Institute, Newcastle University, Baddiley-Clark Building, Richardson Road, \\ Newcastle upon Tyne NE2 4AX, UK \\ 3 ESADE Business School, University Ramon Llull, Avenida de Pedralbes, 60-62, 08034 Barcelona, Spain \\ 4 Institute for Mental Health Policy Research, CAMH, 33 Russell Street, Toronto, ON M5S 2S1, Canada \\ * Correspondence: d.kokole@maastrichtuniversity.nl
}

check for updates

Citation: Kokole, D.; Anderson, P.; Jané-Llopis, E. Nature and Potential Impact of Alcohol Health Warning Labels: A Scoping Review. Nutrients 2021, 13, 3065. https://doi.org/ $10.3390 /$ nu13093065

Academic Editor: Joerg Koenigstorfer

Received: 21 July 2021

Accepted: 27 August 2021

Published: 31 August 2021

Publisher's Note: MDPI stays neutral with regard to jurisdictional claims in published maps and institutional affiliations.

Copyright: (c) 2021 by the authors. Licensee MDPI, Basel, Switzerland. This article is an open access article distributed under the terms and conditions of the Creative Commons Attribution (CC BY) license (https:// creativecommons.org/licenses/by/ $4.0 /)$.

\begin{abstract}
Alcohol is toxic to human health. In addition to providing nutritional information, labels on alcohol products can be used to communicate warnings on alcohol-related harms to consumers. This scoping review examined novel or enhanced health warning labels to assess the current state of the research and the key studied characteristics of labels, along with their impact on the studied outcomes. Four databases (Web of Science, MEDLINE, PsycInfo, CINAHL) were searched between January 2010 and April 2021, and 27 papers were included in the review. The results found that most studies were undertaken in English-speaking populations, with the majority conducted online or in the laboratory setting as opposed to the real world. Seventy percent of the papers included at least one cancer-related message, in most instances referring either to cancer in general or to bowel cancer. Evidence from the only real-world long-term labelling intervention demonstrated that alcohol health warning labels designed to be visible and contain novel and specific information have the potential to be part of an effective labelling strategy. Alcohol health warning labels should be seen as tools to raise awareness on alcohol-related risks, being part of wider alcohol policy approaches.
\end{abstract}

Keywords: alcohol; labelling; health warning labels; effectiveness; implementation

\section{Introduction}

Globally, 5.3\% of all deaths and 5.0\% of all disability adjusted life years are caused by ethanol [1], a toxic substance [2]. Alcohol consumption is causally related to more than 40 ICD-10 three-digit categories [3], including non-communicable diseases such as liver disease [4], cancers of the colon, liver and breast [5-7], alcohol use disorders [8], nonischaemic cardiovascular diseases [9]; communicable diseases such as tuberculosis [10]; and intentional and unintentional injuries [3]. Alcohol can also impair mental health (particularly depression $[11,12]$ ) and cause harm to others, such as in foetal alcohol spectrum disorders $[13,14]$.

Despite alcohol's detrimental effect on a variety of health outcomes, public awareness about some health risks associated with alcohol consumption remains relatively low. A 2018 review of 32 studies found that while awareness of alcohol as a risk factor for cancer varies by country, in most studies, less than half of the respondents correctly identified the alcohol-cancer link [15]. This finding is confirmed in more recent studies [16-18], which additionally found that awareness of the link between alcohol and cancer differed by type of cancer (e.g., the link between alcohol and breast cancer tended to be the least well known).

One of the approaches to raise awareness of the health risks associated with alcohol consumption can be product labelling. A review from 2013 on enhanced labelling [19] 
identified five elements that could be useful to consumers: a list of ingredients, nutritional information, serving size and servings per container, a definition of moderate intake (low risk drinking guidelines) and a health warning label (HWL). While the majority of countries have mandated alcohol volume content on the labels [20], this is not the case with nutritional or health labelling. In many of the high consumption regions, such as EU countries, UK, US, Australia, New Zealand and Canada, alcoholic beverages are exempt from regulations to include nutritional information (for example, nutritional values and calorie content) on the product label [21-24], as opposed to non-alcoholic drinks and food products. Nutritional labelling policy thus leaves much room for improvement, with clear guidelines available on how information should be presented based on the requirements for food and non-alcoholic beverages should nutritional labels be mandated for alcoholic beverages. Whilst there is scarce alcohol-specific research on nutrition information $[25,26]$, much is known from the nutrition field on enhanced presentation of nutritional information [27,28], which could be applied to alcoholic beverages.

There is little available evidence on the effectiveness of health warning labels that would inform consumers about the risks associated with alcohol consumption. Most older studies examining the effectiveness of implemented health warning messages report on the US health warning label, which focuses on risks surrounding drinking during pregnancy, and has not changed since its introduction in 1989 [29,30]. More recent studies on existing health warning labelling schemes, either mandatory [31,32] or voluntary [33-35], show that the existing labels (e.g., pregnancy logo or responsibility message) are suboptimal and are either not being noticed or not being understood. However, evidence from tobacco shows that health warning labels can be a very important part of the broader package of interventions [36,37], and there have been calls to apply tobacco-style health warning labels to alcohol products [38]. Given the evidence for the less effective existing alcohol HWL, this review focuses on examining evidence with regard to novel (not previously implemented in practice) or enhanced labels (improved versions of existing ones).

The importance of labelling is recognised by the World Health Organisation, whose global strategy to reduce the harmful use of alcohol [39] calls for providing consumer information about the harm related to alcohol and labelling alcoholic beverages to indicate such risks. Awareness of such information is, in turn, linked to increased public support for more stringent policies, such as taxation [40,41], and can therefore be an important part of effective alcohol policy. There has been increased interest among policymakers to provide health information to consumers through labels. Ireland passed the Public Health Alcohol Act in 2018, mandating cancer warnings on labels [42] and becoming only the second country in the world after South Korea so to do [20], but nothing else has been implemented as of 2021. There has also been increased research activity, and a number of labelling-related reviews have been published recently. Previous reviews focusing on alcohol HWL operated with a relative scarcity of studies [43], focused only on one aspect of the label (image vs. text) [44] or made a brief narrative overview of health labelling studies as part of broader alcohol labelling [45]. Whilst our work partially overlaps with other reviews in examining the impact of labels, our review adds a thorough and systematic examination of the scope of (quasi-)experimental research on new or enhanced health warning labels, including an overview of the labels used in the studies and the explanatory variables studied. As there is not a single definition of "alcohol $\mathrm{HWL}$ ", labels can differ in how they are developed, implemented and evaluated, and the current review aims to contextualise impacts in light of this information. As health warning labels, we consider labels that are containing information on the relationship between alcohol and health outcomes. We do not focus on drinking guidelines and standard drinks labelling, as this has been carried out elsewhere [46].

Thus, the aim of this scoping review is to examine the peer-reviewed literature studying novel or enhanced health warning labels and to answer two research questions: (1) what is the scope of the current alcohol HWL research (in terms of research aims, studied in- 
dependent variables, the outcomes of interest, as well as label content and format), and (2) what is the impact of the labels and key label characteristics on the studied outcomes?

\section{Methods}

\subsection{Design}

To answer the research questions, we used scoping review methodology, guided by the methodological framework of Arksey and O'Malley [47]. Scoping reviews are used to map the main concepts in the research area and present a broad overview of the existing evidence, including identification of the gaps, regardless of the study quality [48]. The protocol was not published in advance, and the results are reported in accordance with PRISMA-ScR checklist [49].

\subsection{Eligibility Criteria}

To be included in the review, studies had to evaluate new or enhanced alcohol health warning labels in any population. By "new", we considered labels developed for the purpose of the research and not previously implemented in practice, and by "enhanced", we considered improved versions of existing labels already implemented in practice. Studies had to include experimental or quasi-experimental methodology with clearly delineated independent and dependent variables, in order to be able to synthesise results based on the manipulated variables and to identify the key characteristics of effective labels. Only peer-reviewed articles in the English language published in 2010 or after were included, as in the previous reviews, there were no older experimental studies of new messages. Studies that did not include health warning messages, that focused on qualitative data or were only cross-sectional evaluations were excluded.

\subsection{Information Sources and Search Strategy}

Four databases (Web of Science, MEDLINE, PsycInfo, CINAHL) were searched with the abovementioned restrictions on language and dates (English language, January 2010April 2021). Additionally, we complemented the database searches with inspection of reference lists of relevant articles and reviews, and we undertook a Google Scholar search. The search strategy contained a combination of terms related to alcohol labelling: [alcohol AND (label OR label*) AND (message OR information OR warning) AND (experiment* OR eval* OR effect)].

\subsection{Study Selection and Summary}

Study selection was performed by one researcher according to the abovementioned inclusion and exclusion criteria, with ambiguous cases being resolved through consultation with the second researcher. After the removal of duplicates, the studies were first screened by title and abstract, followed by full text examination and final article selection. Next, the data were charted to answer the main research questions. Next to general information on the papers' aim and methodology, information on label development and implementation was charted, as well as the independent studied variables and the results for each of the outcomes. Papers rather than studies were taken as the units of interest, as the focus of the review was to appraise the scope of the published literature and to identify results for all the studied outcomes.

\section{Results}

In total, we identified 27 papers that fit our inclusion criteria (see Appendix A for PRISMA diagram) [50-76]. One paper reported on two studies using different methodologies to study the same variables [50], and four papers reported on different aspects of one intervention [51-54]. The remaining papers were individual studies (with a complete or partial focus on alcohol HWL labelling), although some of them were part of the same research line of label development and testing (e.g., [55-60]) or studied the same labels (e.g., [61,62]). 
Table 1 provides an overview of the papers with their aim and methodology. Twothirds (18) of the included papers were published in 2018 or later, indicating an increasing research interest in the topic. All of the research was undertaken in Western countries: most papers reported on research conducted in the UK (nine), Canada (six) and Australia (five). The remaining papers reported on research conducted in the US (three), Germany (two) or were multi-country (twice Luxembourg/Germany, and once Italy/France). Only four papers described real-world interventions. The remainder of the studies were either conducted online (sixteen) or in a laboratory (eight, of which one was in a naturalistic shopping laboratory). Six studies had under 100 participants, eleven studies had above 1000 participants and one study relied on sales data. The majority $(80 \%)$ of the studies with a lower number of participants were conducted in the laboratory and before 2018, indicating that in recent years, larger sample sizes were accessible in an easier manner through online panels. Two-thirds of the papers focused on the general adult (of legal drinking age in the country) drinking population, although the exact inclusion criteria differed and ranged from people drinking at least once in the past 12 months, to people consuming alcohol weekly. Eight of the papers focused on the younger population/students (some by convenience rather than by inclusion criteria) and one paper focused on secondary school students.

\subsection{Research Scope}

To answer the first research question, we looked at the aim of the papers and the variables studied (both independent variables and the outcomes), as well as at the content and format of the studied labels.

In terms of study aims and studied independent variables, five papers (19\%) compared only new or enhanced labels with no or regular labels [51-54,63]. Focusing on label content, eight papers (30\%) compared text-only labels with text and image labels $[55,56,61,62,64-67]$, twelve studies (44\%) compared text message characteristics (e.g., content, specificity, framing, source, use of causal language) [57-60,67-74] and three (11\%) compared image characteristics $[57,66,75]$. Four (15\%) papers compared label formats, such as size, position, colour or branding $[50,64,70,76]$. Additionally, nine papers $(33 \%)$ studied other variables, such as alcohol content, alcohol/substance type, efficacy information, expectancy and self-affirmation [64-66,68,69,73-76].

The majority of papers investigated more than one outcome (Table 2). The most commonly studied outcomes were attitude ( $41 \%$ of the papers), intentions (30\%), emotionrelated outcomes (e.g., fear, worry) (26\%) and acceptability/support for the labels (26\%). Behaviour was studied in six papers (22\%) — in three of them through self-report [51,54,67], but also through purchasing [53,56] or consuming alcohol [62].

The tested labels were predominantly developed by the researchers themselves, based either on other research (e.g., related to tobacco and alcohol labels, alcohol harm, effective messages) or on own development and piloting (e.g., through qualitative studies), or both. In the four papers related to a real-world intervention [51-54], the labels were additionally (next to own and other research) developed in consultation with local stakeholders. In two cases, the labels used in the research were developed by a non-governmental organisation [67,75], and in one case [50], existing voluntary labels were enhanced based on theory. 
Table 1. Studies overview-aim and methodology.

\begin{tabular}{|c|c|c|c|c|c|c|c|c|}
\hline $\begin{array}{l}\text { Authors and Year } \\
\text { Published }\end{array}$ & Country & $\begin{array}{c}\text { Study Aim } \\
\text { (According to Authors) }\end{array}$ & Setting & $\begin{array}{c}\text { Methodology/Study } \\
\text { Desiggn }\end{array}$ & Sample Characteristics & $\begin{array}{c}\text { Population (P) or Inclusion } \\
\text { Criteria (IC) }\end{array}$ & Sampling and Recruitment & $\begin{array}{l}\text { Year Data } \\
\text { Collected }\end{array}$ \\
\hline $\begin{array}{l}\text { Al-Hamdani and } \\
\text { Smith (2015) [64] }\end{array}$ & Canada & $\begin{array}{l}\text { To apply the lessons learned from the tobacco } \\
\text { health warnings and plain packaging } \\
\text { literature to an alcohol packaging study and } \\
\text { test whether labelling alters } \\
\text { consumer perceptions. }\end{array}$ & Online (survey) & $\begin{array}{l}\text { Experiment: } 3 \times 4 \\
\text { mixed design }\end{array}$ & $\begin{array}{c}\mathrm{N}=92 \\
\mathrm{M}(\mathrm{SD})_{\mathrm{age}}=36.4(13.3) \\
66.2 \% \text { female }\end{array}$ & $\begin{array}{l}\text { P: } 60.2 \% \text { students, } 39.8 \% \\
\text { hospital employees } \\
91.1 \% \text { participants drinking } \\
\text { at least weekly }\end{array}$ & $\begin{array}{l}\text { Convenience sample, recruited } \\
\text { through posters and ads in } \\
\text { the organisations }\end{array}$ & $\begin{array}{l}\text { Not } \\
\text { reported }\end{array}$ \\
\hline $\begin{array}{l}\text { Al-Hamdani and } \\
\text { Smith (2017) [76] }\end{array}$ & Canada & $\begin{array}{l}\text { To examine whether increasing the size of } \\
\text { HWL and plain packaging lowers ratings of } \\
\text { alcohol products and the consumers who use } \\
\text { them, increases ratings of bottle "boringness" } \\
\text { and enhances warning recognition compared } \\
\text { with branded packaging. }\end{array}$ & Online (survey) & $\begin{array}{l}\text { Experiment: } 3 \times 2 \times 3 \\
\text { mixed design }\end{array}$ & $\begin{array}{l}\mathrm{N}=440 \text { initially } / 241 \\
\quad \text { inally } \\
\mathrm{M}(\mathrm{SD})_{\mathrm{age}}=26(7.1) \\
51.7 \% \text { female }\end{array}$ & $\begin{array}{l}\text { IC: Adults of legal age who } \\
\text { consumed alcohol in the } \\
\text { past } 12 \text { months } \\
\text { P: } 91 \% \text { participants drinking } \\
\text { at least weekly }\end{array}$ & $\begin{array}{l}\text { Convenience sample, } \\
\text { recruited online }\end{array}$ & $\begin{array}{c}\text { Not } \\
\text { reported }\end{array}$ \\
\hline $\begin{array}{l}\text { Annunziata et al. } \\
\text { (2019) [70] }\end{array}$ & $\begin{array}{l}\text { Italy and } \\
\text { France }\end{array}$ & $\begin{array}{c}\text { To analyse Generation Y consumers' } \\
\text { preferences for, interest in and attitudes } \\
\text { towards different formats of health warnings } \\
\text { on wine labels in two countries with different } \\
\text { legal approaches: France and Italy. }\end{array}$ & Online (survey) & $\begin{array}{l}\text { Discrete choice } \\
\text { experiment }\end{array}$ & $\begin{array}{c}\mathrm{N}=500(250 \text { per country }) \\
\mathrm{M}(\mathrm{SD})_{\text {age }}=23.3(3.4)-\mathrm{FR} ; \\
25.2(4.5)-\mathrm{IT} \\
54 \% \text { females-FR; } 60 \% \\
\text { females-IT }\end{array}$ & $\begin{array}{l}\text { IC: Generation Y } \\
\quad(1978-2000)\end{array}$ & $\begin{array}{l}\text { Convenience sample, } \\
\text { recruited online }\end{array}$ & 2018 \\
\hline $\begin{array}{l}\text { Blackwell et al. } \\
\text { (2018) [71] }\end{array}$ & UK & $\begin{array}{l}\text { To examine the influence of unit labels and } \\
\text { health warnings on drinkers' understanding, } \\
\text { attitudes and behavioural intentions } \\
\text { regarding drinking and examine optimal } \\
\text { methods of delivering this information } \\
\text { on labels. }\end{array}$ & Online (survey) & $\begin{array}{l}\text { Between subjects } \\
\text { experimental study }\end{array}$ & $\begin{array}{c}\mathrm{N}=1184 \\
\mathrm{M}(\mathrm{SD}) \text { age }=35(12) \\
50 \% \text { female }\end{array}$ & $\begin{array}{l}\text { IC: Adult (18+) } \\
\text { drinkers only }\end{array}$ & $\begin{array}{l}\text { Recruitment through } \\
\text { crowdsourcing } \\
\text { platform/panel }\end{array}$ & $\begin{array}{c}\text { Not } \\
\text { reported }\end{array}$ \\
\hline $\begin{array}{l}\text { Clarke, Pechey } \\
\text { et al. (2021a) [55] }\end{array}$ & UK & $\begin{array}{l}\text { To obtain a preliminary assessment of the } \\
\text { possible impact of (i) image-and-text, (ii) text } \\
\text { only and (iii) image-only HWLs on selection } \\
\text { of alcoholic versus non-alcoholic drinks. }\end{array}$ & Online (survey) & $\begin{array}{l}\text { Between-subjects } \\
\text { experimental study, } \\
2 \times 2 \text { factorial design }\end{array}$ & $\begin{array}{c}\mathrm{N}=6024 \text { (completed } \\
\text { the study) } \\
\mathrm{M}(\mathrm{SD}) \text { age }=49.5(15.5) 50 \% \\
\text { female }\end{array}$ & $\begin{array}{l}\text { IC: Adults (18+) who } \\
\text { consumed beer or wine } \\
\text { regularly (i.e., at least once } \\
\text { a week) }\end{array}$ & $\begin{array}{l}\text { Recruited via market } \\
\text { research agency }\end{array}$ & 2019 \\
\hline $\begin{array}{l}\text { Clarke, Blackwell } \\
\text { et al. (2021b) [56] }\end{array}$ & UK & $\begin{array}{l}\text { To estimate the impact of HWLs describing } \\
\text { adverse health consequences of excessive } \\
\text { alcohol consumption on selection of } \\
\text { alcoholic drinks. }\end{array}$ & $\begin{array}{l}\text { Naturalistic } \\
\text { shopping } \\
\text { laboratory }\end{array}$ & $\begin{array}{l}\text { Between-subjects } \\
\text { design }\end{array}$ & $\begin{array}{c}\mathrm{N}=399 \\
\mathrm{M}(\mathrm{SD})_{\text {age }}=39.9(13.7) 55 \% \\
\text { female }\end{array}$ & $\begin{array}{l}\text { IC: Adults (18+) who } \\
\text { purchased beer or wine } \\
\text { weekly to drink at home }\end{array}$ & $\begin{array}{l}\text { Recruited via market } \\
\text { research agency }\end{array}$ & 2020 \\
\hline $\begin{array}{l}\text { Glock and } \\
\text { Krolak-Schwerdt } \\
\text { (2013) [72] }\end{array}$ & $\begin{array}{l}\text { Luxembourg } \\
\text { and Germany }\end{array}$ & $\begin{array}{l}\text { Compared the effectiveness of warning labels } \\
\text { that contradicted positive outcome } \\
\text { expectancies with health-related } \\
\text { warning labels. }\end{array}$ & Laboratory & $\begin{array}{l}\text { Between-subjects } \\
\text { experimental study, } \\
\text { two factorial } \\
\text { mixed design }\end{array}$ & $\begin{array}{c}\mathrm{N}=40 \\
\mathrm{M}(\mathrm{SD})_{\mathrm{age}}=24.0(3.2) \\
60 \% \text { female }\end{array}$ & $\begin{array}{l}\text { P: Undergraduates, native } \\
\text { German speakers } \\
95 \% \text { drinkers }\end{array}$ & $\begin{array}{l}\text { Recruited through } \\
\text { university courses }\end{array}$ & $\begin{array}{c}\text { Not } \\
\text { reported }\end{array}$ \\
\hline $\begin{array}{l}\text { Gold et al. } \\
\text { (2020) [63] }\end{array}$ & UK & $\begin{array}{l}\text { To examine whether showing people a health } \\
\text { warning alongside our label designs would } \\
\text { have a further effect on our secondary } \\
\text { outcomes, increasing the perceived risk of } \\
\text { alcohol consumption, decreasing the } \\
\text { motivation to drink and lowering the level of } \\
\text { drinking which people believe to be } \\
\text { health-damaging. }\end{array}$ & Online (survey) & $\begin{array}{c}\text { Parallel } \\
\text { randomisedcontrolled } \\
\text { trial }\end{array}$ & $\begin{array}{c}\mathrm{N}=7516 \text { total } / 500 \\
\quad \text { for HWL } \\
\mathrm{M}(\mathrm{SD})_{\text {age }}=44.2 \\
(16.5) 50.5 \% \text { female }\end{array}$ & $\begin{array}{l}\text { IC: English adults (18+) } \\
\text { reporting drinking alcohol }\end{array}$ & $\begin{array}{l}\text { Representative sample of the } \\
\text { adult population of Englandin } \\
\text { terms of age, gender and } \\
\text { region, recruited through } \\
\text { online panel platform }\end{array}$ & 2019 \\
\hline $\begin{array}{l}\text { Hall et al. } \\
\text { (2019) [73] }\end{array}$ & US & $\begin{array}{l}\text { To examine US adults' reactions to health } \\
\text { warnings with strong versus weak } \\
\text { causal language. }\end{array}$ & Online (survey) & $\begin{array}{l}\text { Between-subjects } \\
\text { experimental study }\end{array}$ & $\begin{array}{c}\mathrm{N}=1360 \\
\mathrm{M}(\mathrm{SD})_{\text {age }}=37.4(11.6) \\
47.3 \% \text { female }\end{array}$ & IC: US residents, $(18+)$ & $\begin{array}{l}\text { Convenience sample, } \\
\text { recruitment via online } \\
\text { crowdsourcing platform }\end{array}$ & 2018 \\
\hline
\end{tabular}


Table 1. Cont.

\begin{tabular}{|c|c|c|c|c|c|c|c|c|}
\hline $\begin{array}{l}\text { Authors and Year } \\
\text { Published }\end{array}$ & Country & $\begin{array}{c}\text { Study Aim } \\
\text { (According to Authors) }\end{array}$ & Setting & $\begin{array}{c}\text { Methodology/Study } \\
\text { Design }\end{array}$ & Sample Characteristics & $\begin{array}{c}\text { Population (P) or Inclusion } \\
\text { Criteria (IC) }\end{array}$ & Sampling and Recruitment & $\begin{array}{l}\text { Year Data } \\
\text { Collected }\end{array}$ \\
\hline $\begin{array}{l}\text { Hall et al. } \\
\text { (2020) [65] }\end{array}$ & US & $\begin{array}{l}\text { To examine reactions to graphic versus } \\
\text { text-only warnings for cigarettes, SSBs } \\
\text { and alcohol. }\end{array}$ & Online (survey) & $\begin{array}{c}2 \times 2 \times 3 \\
\text { Between-subjects } \\
\text { experimental study }\end{array}$ & $\begin{array}{c}\mathrm{N}=1352 \\
\mathrm{M}_{\mathrm{age}}=37 \\
47 \% \text { female }\end{array}$ & IC: US residents, (18+) & $\begin{array}{l}\text { Convenience sample, } \\
\text { recruitment via online } \\
\text { crowdsourcing platform }\end{array}$ & 2018 \\
\hline $\begin{array}{l}\text { Hobin, Schoueri- } \\
\text { Mychasiw et al. } \\
\text { (2020a) [51] }\end{array}$ & Canada & $\begin{array}{l}\text { To examine the effects of strengthening } \\
\text { alcohol labels on consumer attention and } \\
\text { message processing, and a self-reported } \\
\text { reduction in drinking due to the labels. }\end{array}$ & Real-world & $\begin{array}{l}\text { Quasi } \\
\text { experimental design }\end{array}$ & $\begin{array}{c}\mathrm{N}=2049 \text { unique cohort } \\
\text { participants } \\
\mathrm{M}(\mathrm{SD})_{\text {age }}=\text { intervention } \\
47.4 \text { (14.6); comparison } \\
41.2 \text { (13.7) } \\
\text { 50.7\% female intervention, } \\
45.1 \% \text { female control }\end{array}$ & $\begin{array}{l}\text { IC: Adult (19+), current } \\
\text { drinkers (at least } 1 \text { drink in } \\
\text { past } 30 \text { days), living in the } \\
\text { intervention or comparison } \\
\text { cities, bought alcohol at the } \\
\text { liquor store and did not } \\
\text { self-report being pregnant } \\
\text { or breast-feeding }\end{array}$ & $\begin{array}{c}\text { Systematic recruitment } \\
\text {-standard intercept technique } \\
\text { of approaching every person } \\
\text { that passed a } \\
\text { pre-identified landmark }\end{array}$ & $2017 / 2018$ \\
\hline $\begin{array}{l}\text { Hobin, } \\
\text { Weerasinghe et al. } \\
\text { (2020b) [52] }\end{array}$ & Canada & $\begin{array}{l}\text { To test the initial and continued effects of } \\
\text { cancer warning labels on drinkers' recall and } \\
\text { knowledge that alcohol can cause cancer. }\end{array}$ & Real-world & $\begin{array}{l}\text { Quasi } \\
\text { experimental design }\end{array}$ & $\begin{array}{c}\mathrm{N}=2049 \text { unique } \\
\text { cohort participants } \\
\mathrm{M}(\mathrm{SD})_{\text {age }}=\text { intervention } \\
47.4(14.6) ; \text {; comparison } \\
41.2(13.7) \\
50.7 \% \text { female intervention, } \\
45.1 \% \text { female control }\end{array}$ & $\begin{array}{l}\text { IC: Adult (19+) current } \\
\text { drinkers (at least } 1 \text { drink in } \\
\text { past } 30 \text { days), living in the } \\
\text { intervention or comparison } \\
\text { cities, bought alcohol at the } \\
\text { liquor store, did not } \\
\text { self-report being pregnant } \\
\text { or breast-feeding }\end{array}$ & $\begin{array}{c}\text { Systematic } \\
\text { recruitment-standard } \\
\text { intercept technique of } \\
\text { approaching every person that } \\
\text { passed a } \\
\text { pre-identified landmark }\end{array}$ & $2017 / 2018$ \\
\hline $\begin{array}{l}\text { Hobin, Shokar } \\
\text { et al. (2020c) [54] }\end{array}$ & Canada & $\begin{array}{l}\text { To investigate the impact of alcohol labels on } \\
\text { (i) unprompted recall of label messages, (ii) } \\
\text { depth of cognitive processing of label } \\
\text { messages and (iii) self-reported impact on } \\
\text { alcohol consumption. }\end{array}$ & Real-world & $\begin{array}{l}\text { Quasi } \\
\text { experimental design }\end{array}$ & $\begin{array}{c}\mathrm{N}=1647 \text { unique cohort } \\
\text { participants } \\
\text { Age: Intervention: } 6.8 \% \\
19-24,34.3 \% \text { 25 } 25-44,58.8 \% \\
45+ \\
\text { Control } 12.3 \% 19-24, \\
45.5 \% 25-44,42.2 \% 45+ \\
51.5 \% \text { female intervention, } \\
44.1 \% \text { female control }\end{array}$ & $\begin{array}{l}\text { IC: Adult (19+) current } \\
\text { drinkers (at least } 1 \text { drink in } \\
\text { past } 30 \text { days), living in the } \\
\text { intervention or comparison } \\
\text { cities, bought alcohol at the } \\
\text { liquor store, did not } \\
\text { self-report being pregnant } \\
\text { or breast-feeding }\end{array}$ & $\begin{array}{c}\text { Systematic } \\
\text { recruitment-standard } \\
\text { intercept technique of } \\
\text { approaching every person that } \\
\text { passed a } \\
\text { pre-identified landmark }\end{array}$ & $2017 / 2018$ \\
\hline $\begin{array}{l}\text { Jarvis and } \\
\text { Pettigrew } \\
\text { (2013) [74] }\end{array}$ & Australia & $\begin{array}{l}\text { To assess the effects of warning statements on } \\
\text { youth's alcohol purchase decisions in the } \\
\text { context of information relating to proprietary } \\
\text { brands and alcohol content. }\end{array}$ & Online (survey) & $\begin{array}{l}\text { Discrete choice } \\
\text { experiment }\end{array}$ & $\begin{array}{l}\mathrm{N}=300 \\
\text { Age and gender } \\
\text { not reported }\end{array}$ & $\begin{array}{l}\text { IC: } 18-25 \text { year old current } \\
\text { drinkers of pre-mixed } \\
\text { alcoholic beverages, } \\
\text { purchased a packaged } \\
\text { pre-mixed alcoholic } \\
\text { beverage in the past the } \\
4 \text { weeks }\end{array}$ & $\begin{array}{l}\text { Through web panel provider, } \\
\text { including } \\
\text { equal proportional } \\
\text { representation of two } \\
\text { age categories }\end{array}$ & $\begin{array}{l}\text { Not } \\
\text { reported }\end{array}$ \\
\hline $\begin{array}{l}\text { Jongenelis, } \\
\text { Pettigrew et al. } \\
\text { (2018a) [58] }\end{array}$ & Australia & $\begin{array}{l}\text { To examine the effectiveness of messages } \\
\text { when delivered } \\
\text { by single versus multiple sources. }\end{array}$ & Online (survey) & $\begin{array}{l}\text { Between-subjects } \\
\text { experimental study }\end{array}$ & $\begin{array}{c}\mathrm{N}=2087 \\
\mathrm{M}(\mathrm{SD})_{\mathrm{age}}=36.05(12.67) \\
50.1 \% \text { female }\end{array}$ & $\begin{array}{l}\text { IC: Adult drinkers } \\
\text { consuming alcohol at least } \\
\text { twice per month }\end{array}$ & Web panel provider & $\begin{array}{l}\text { Not } \\
\text { reported }\end{array}$ \\
\hline $\begin{array}{l}\text { Jongenelis, Pratt } \\
\text { et al. (2018b) [59] }\end{array}$ & Australia & $\begin{array}{l}\text { To examine whether exposing at-risk drinkers } \\
\text { to warning statements relating to specific } \\
\text { chronic diseases increases the extent to which } \\
\text { alcohol is believed to be a risk factor for those } \\
\text { diseases and influences } \\
\text { consumption intentions. }\end{array}$ & Online (survey) & $\begin{array}{l}\text { Between-subjects } \\
\text { experimental study }\end{array}$ & $\begin{array}{l}N=364 \\
18-34 \text { years } 29 \% \\
35-65 \text { years } 71 \% \\
28 \% \text { female }\end{array}$ & $\begin{array}{l}\text { IC: Adults } 18-65 \text { years who } \\
\text { reported drinking at levels } \\
\text { associated with long-term } \\
\text { risk of harm (more than } 2 \\
\text { SD per day) }\end{array}$ & Web panel provider & $\begin{array}{l}\text { Not } \\
\text { reported }\end{array}$ \\
\hline $\begin{array}{l}\text { Krischler and } \\
\text { Glock (2015) [68] }\end{array}$ & $\begin{array}{l}\text { Luxembourg } \\
\text { and Germany }\end{array}$ & $\begin{array}{l}\text { To investigate the effectiveness of alcohol } \\
\text { warning labels tailored toward young adults' } \\
\text { positive outcome expectancies. }\end{array}$ & Laboratory & $\begin{array}{l}\text { Experiment: } 3 \times 2 \\
\text { mixed design }\end{array}$ & $\begin{array}{c}\mathrm{N}=122 \\
\mathrm{M}(\mathrm{SD}))_{\text {age }}=23.5(3.5) \\
68.9 \% \text { female }\end{array}$ & P: 91.7\% Undergraduates & Recruited on campus & 2014 \\
\hline
\end{tabular}


Table 1. Cont.

\begin{tabular}{|c|c|c|c|c|c|c|c|c|}
\hline $\begin{array}{l}\text { Authors and Year } \\
\text { Published }\end{array}$ & Country & $\begin{array}{c}\text { Study Aim } \\
\text { (According to Authors) }\end{array}$ & Setting & $\begin{array}{c}\text { Methodology/Study } \\
\text { Design }\end{array}$ & Sample Characteristics & $\begin{array}{c}\text { Population (P) or Inclusion } \\
\text { Criteria (IC) }\end{array}$ & Sampling and Recruitment & $\begin{array}{l}\text { Year Data } \\
\text { Collected }\end{array}$ \\
\hline Ma (2021) [69] & US & $\begin{array}{l}\text { To determine the impact of pictorial warning } \\
\text { labels featuring narrative content on risk } \\
\text { perceptions and behavioural intentions. }\end{array}$ & Online (survey) & $\begin{array}{l}\text { Between-subjects } \\
\text { experimental study }\end{array}$ & $\begin{array}{c}\mathrm{N}=169 \\
\mathrm{M}(\mathrm{SD})_{\mathrm{age}}=43.2(11.5) \\
37.9 \% \text { female }\end{array}$ & IC: Alcohol consumers & $\begin{array}{l}\text { Recruited through web } \\
\text { panel provider }\end{array}$ & $\begin{array}{l}\text { Not } \\
\text { reported }\end{array}$ \\
\hline $\begin{array}{l}\text { Monk et al. } \\
\text { (2017) [66] }\end{array}$ & UK & $\begin{array}{l}\text { To investigate the amount of time spent } \\
\text { looking at the different elements of } \\
\text { alcohol-related health warnings. }\end{array}$ & Laboratory & $\begin{array}{l}\text { Experiment: } 2 \times 2 \times 2 \\
\text { mixed factorial design }\end{array}$ & $\begin{aligned} \mathrm{N} & =22 \\
\mathrm{M}(\mathrm{SD})_{\mathrm{age}} & =21.3(1.7) \\
68.2 \% \text { female } & \end{aligned}$ & University students & Opportunity sampling & $\begin{array}{l}\text { Not } \\
\text { reported }\end{array}$ \\
\hline $\begin{array}{l}\text { Morgenstern et al. } \\
\quad(2021)[67]\end{array}$ & Germany & $\begin{array}{l}\text { To investigate impact of alcohol warning } \\
\text { labels on knowledge and negative emotions. }\end{array}$ & Online (survey) & $\begin{array}{l}\text { Three factorial } \\
\text { experiment }\end{array}$ & $\begin{array}{c}\mathrm{N}=9260 \\
\mathrm{M}(\mathrm{SD})_{\mathrm{agg}}=12.9(1.8) \\
48.6 \% \text { female }\end{array}$ & $\begin{array}{l}\text { IC: Secondary school } \\
\text { students (10-17) }\end{array}$ & $\begin{array}{l}\text { Recruited through schools } \\
\text { from randomly selected } \\
\text { sub-regions }\end{array}$ & 2017-2018 \\
\hline $\begin{array}{l}\text { Pettigrew et al. } \\
\text { (2016) [60] }\end{array}$ & Australia & $\begin{array}{l}\text { To investigate the potential effectiveness of } \\
\text { alcohol warning statements designed to } \\
\text { increase awareness of the alcohol-cancer link. }\end{array}$ & Online (survey) & $\begin{array}{l}\text { Between-subjects } \\
\text { experimental study }\end{array}$ & $\begin{array}{c}\mathrm{N}=1680 \\
<31 \text { years } 49.5 \\
31-45 \text { years } 25.2 \\
46-65 \text { years } 25.3 \\
49.9 \% \text { female }\end{array}$ & $\begin{array}{l}\text { IC: Adult (18-65) drinkers, } \\
\text { consuming alcohol at least } \\
\text { two days per month }\end{array}$ & Web panel provider & $\begin{array}{l}\text { Not } \\
\text { reported }\end{array}$ \\
\hline $\begin{array}{l}\text { Pham et al. } \\
(2018)^{*}[50]\end{array}$ & Australia & $\begin{array}{l}\text { To investigate attention of current in market } \\
\text { alcohol warning labels and examine whether } \\
\text { attention can be enhanced through } \\
\text { theoretically informed design. }\end{array}$ & $\begin{array}{l}\text { Study 1: Online } \\
\text { (survey) } \\
\text { Study 2: } \\
\text { Laboratory }\end{array}$ & $\begin{array}{l}\text { Between-subjects } \\
\text { experimental study }\end{array}$ & $\begin{array}{c}\text { Study 1: } \mathrm{N}=559 \\
\text { M(SD) age }=31.9(7.8) \\
\text { Gender not reported } \\
\text { Study 2: } \mathrm{N}=87 \\
\text { M(SD) age }=26.6 \\
\text { (10.5)Gender not reported }\end{array}$ & $\begin{array}{l}\text { Study 1: P: } 72 \% \text { employed, } \\
\text { 6.6\% full time students } \\
\text { Study 2: P } 49.4 \% \text { full time } \\
\text { students, } 41.4 \% \text { employed }\end{array}$ & $\begin{array}{l}\text { Study 1: Snowball } \\
\text { recruitment online } \\
\text { Study 2: Face to face } \\
\text { on campus }\end{array}$ & 2015 \\
\hline $\begin{array}{l}\text { Sillero-Rejon et al. } \\
\quad \text { (2018) [75] }\end{array}$ & UK & $\begin{array}{c}\text { To examine whether enhancing } \\
\text { self-affirmation among a population of } \\
\text { drinkers, prior to viewing threatening alcohol } \\
\text { pictorial health warning labels, would reduce } \\
\text { defensive reactions and promote reactions } \\
\text { related to behaviour change, and whether } \\
\text { there is an interaction between } \\
\text { self-affirmation and severity of warning. }\end{array}$ & Laboratory & $\begin{array}{l}\text { Between-subjects } \\
\text { experimental study }\end{array}$ & $\begin{array}{c}\mathrm{N}=128 \\
\mathrm{M}(\mathrm{SD}))_{\text {age }}=22(4) \\
\text { Gender not reported }\end{array}$ & $\begin{array}{l}\text { IC: Adult }(18+) \text { regular } \\
\text { alcohol consumers who } \\
\text { have consumed over } 14 \\
\text { units per week during the } \\
\text { preceding week }\end{array}$ & $\begin{array}{l}\text { Opportunity sampling, } \\
\text { recruited via e-mail, posters } \\
\text { and websites }\end{array}$ & $\begin{array}{l}\text { Not } \\
\text { reported }\end{array}$ \\
\hline $\begin{array}{l}\text { Stafford and } \\
\text { Salmon } \\
(2017)[62]\end{array}$ & UK & $\begin{array}{l}\text { To test whether the speed of alcohol } \\
\text { consumption is influenced by the type of } \\
\text { alcohol health warning contained } \\
\text { on the beverage. }\end{array}$ & Laboratory & $\begin{array}{l}\text { Between-subjects } \\
\text { experimental study }\end{array}$ & $\begin{array}{c}\mathrm{N}=45 \\
\mathrm{M}(\mathrm{SD})_{\mathrm{age}}=18.9(1.1) \\
100 \% \text { female }\end{array}$ & $\begin{array}{l}\text { IC: Female (18-25) regular } \\
\text { alcohol consumers }\end{array}$ & $\begin{array}{l}\text { Recruited using an online } \\
\text { system } \\
\text { and via social media }\end{array}$ & $\begin{array}{l}\text { Not } \\
\text { reported }\end{array}$ \\
\hline $\begin{array}{l}\text { Wigg and } \\
\text { Stafford } \\
(2016)[61]\end{array}$ & UK & $\begin{array}{l}\text { To test the effectiveness of a range of alcohol } \\
\text { health warnings, comparing no health } \\
\text { warning, text only and pictorial warning. }\end{array}$ & Laboratory & $\begin{array}{l}\text { Between-subjects } \\
\text { experimental study }\end{array}$ & $\begin{array}{c}\mathrm{N}=60 \\
\mathrm{M}(\mathrm{SD}) \text { age }=19.4(3.1) \\
71.7 \% \text { female }\end{array}$ & IC: Alcohol consumers & $\begin{array}{l}\text { Recruited using an online } \\
\text { system, received credit points }\end{array}$ & $\begin{array}{l}\text { Not } \\
\text { reported }\end{array}$ \\
\hline $\begin{array}{l}\text { Zhao et al. } \\
\text { (2020) [53] }\end{array}$ & Canada & $\begin{array}{l}\text { To test if the labelling intervention was } \\
\text { associated with reduced } \\
\text { alcohol consumption. }\end{array}$ & Real-world & $\begin{array}{l}\text { Quasi } \\
\text { experimental design }\end{array}$ & $\begin{array}{l}\text { Monthly retail sales data, } \\
\text { no individual participants }\end{array}$ & $\begin{array}{l}\text { Population } 15+\text { in the } \\
\text { research areas }\end{array}$ & I & $2017 / 2018$ \\
\hline
\end{tabular}

* two studies considered as one, as they are studying the same outcome. 
Table 2. Studied outcomes.

\begin{tabular}{|c|c|c|c|}
\hline & $\mathbf{N}$ & $\%$ & Papers \\
\hline Attitude & 11 & $41 \%$ & $\begin{array}{c}\text { Al-Hamdani \& Smith (2015) [64]; Al-Hamdani \& Smith (2017) [76]; } \\
\text { Annunziata et al. (2019) [70]; Blackwell et al. (2018) [71]; Glock \& } \\
\text { Krolak-Schwerdt (2013) [72]; Hall et al. (2020) [65]; Jarvis \& } \\
\text { Pettigrew (2013) [74]; Jongenelis et al. (2018a) [58]; Krischler \& } \\
\text { Glock (2015) [68]; Pettigrew et al. (2016) [60]; Stafford \& Salmon } \\
\text { (2017) [62] }\end{array}$ \\
\hline Intentions & 8 & $30 \%$ & $\begin{array}{l}\text { Clarke et al. (2021a) [55]; Glock \& Krolak-Schwerdt (2013) [72]; } \\
\text { Jongenelis et al. (2018a) [58]; Jongenelis et al. (2018b) [59]; } \\
\text { Krischler \& Glock (2015) [68]; Ma (2021) [69]; Pettigrew et al. } \\
\text { (2016) [60]; Wigg \& Stafford (2016) [61] }\end{array}$ \\
\hline Emotion & 7 & $26 \%$ & $\begin{array}{l}\text { Clarke et al. (2021a) [55]; Clarke et al. (2021b) [56]; Hall et al. } \\
\text { (2020) [65]; Ma (2021) [69]; Morgenstern et al. (2021) [67]; } \\
\text { Pechey et al. (2020) [57]; Wigg \& Stafford (2016) [61] }\end{array}$ \\
\hline Acceptability/support & 7 & $26 \%$ & $\begin{array}{l}\text { Blackwell et al. (2018) [71]; Clarke et al. (2021a) [55]; Clarke et al. } \\
\text { (2021b) [56]; Hall et al. (2020) [65]; Hall et al. (2019) [73] } \\
\text { Hobin et al. (2020b) [52]; Pechey et al. (2020) [57] }\end{array}$ \\
\hline Behaviour & 6 & $22 \%$ & $\begin{array}{l}\text { Clarke et al. (2021b) [56]; Hobin et al. (2020a) [51]; Hobin et al. } \\
\text { (2020c) [54]; Morgenstern et al. (2021) [67]; Stafford \& Salmon } \\
\text { (2017) [62]; Zhao et al. (2020) [53] }\end{array}$ \\
\hline Awareness/recognition & 5 & $19 \%$ & $\begin{array}{c}\text { Al-Hamdani \& Smith (2015) [64]; Al-Hamdani \& Smith (2017) [76]; } \\
\text { Hobin et al. (2020a) [51]; Hobin et al. (2020b) [52]; } \\
\text { Hobin et al. (2020c) [54] }\end{array}$ \\
\hline Risk perception & 5 & $19 \%$ & $\begin{array}{l}\text { Clarke et al. (2021a) [55]; Gold et al. (2020) [63]; Ma (2021) [69]; } \\
\text { Sillero-Rejon et al. (2018) [75]; Wigg \& Stafford (2016) [61] }\end{array}$ \\
\hline Motivation & 4 & $15 \%$ & $\begin{array}{l}\text { Blackwell et al. (2018) [71]; Gold et al. (2020) [63]; Pechey et al. } \\
\text { (2020) [57]; Sillero-Rejon et al. (2018) [75] }\end{array}$ \\
\hline Reactance & 4 & $15 \%$ & $\begin{array}{l}\text { Blackwell et al. (2018) [71]; Clarke et al. (2021a) [55]; Hall et al. } \\
\text { (2020) [65]; Sillero-Rejon et al. (2018) [75] }\end{array}$ \\
\hline Knowledge & 3 & $11 \%$ & $\begin{array}{c}\text { Hobin et al. (2020b) [52]; Jongenelis et al. (2018b) [59]; } \\
\text { Morgenstern et al. (2021) [67] }\end{array}$ \\
\hline Perceived effectiveness & 3 & $11 \%$ & $\begin{array}{l}\text { Hall et al. (2020) [65]; Hall et al. (2019) [73]; } \\
\text { Sillero-Rejon et al. (2018) [75] }\end{array}$ \\
\hline Attention & 3 & $11 \%$ & $\begin{array}{l}\text { Monk et al. (2017) [66]; Pham et al. (2018) [50]; } \\
\text { Sillero-Rejon et al. (2018) [75] }\end{array}$ \\
\hline Cognitive processing & 3 & $11 \%$ & $\begin{array}{l}\text { Hall et al. (2020) [65]; Hobin et al. (2020a) [51]; } \\
\text { Hobin et al. (2020c) [54] }\end{array}$ \\
\hline Avoidance & 3 & $11 \%$ & $\begin{array}{c}\text { Blackwell et al. (2018) [71]; Clarke et al. (2021a) [55]; } \\
\text { Sillero-Rejon et al. (2018) [75] }\end{array}$ \\
\hline Efficacy & 2 & $7 \%$ & Blackwell et al. (2018) [71]; Hall et al. (2020) [65] \\
\hline
\end{tabular}

In terms of label content, eight studies studied a single message; the remaining were either comparing different labels (or groups of labels) or used several messages as a single intervention. Most commonly, the messages were referring either to cancer in general (e.g., "Warning: Alcohol causes cancer") [51-54,57-60,63,67,71,75], or specifically to bowel cancer (e.g., "Alcohol causes bowel cancer") [51-60,69,71], both in $41 \%$ of all papers. Overall, $70 \%$ of papers included at least one cancer-related message, with other mentioned cancers being also breast (33\%) [51-58,60] and liver cancer $(30 \%)[55-57,61,62,64,69,76]$. Liver cirrhosis or liver disease message was used in nine (33\%) papers (e.g., "Warning: Alcohol causes liver disease $\left.^{\prime \prime}\right)([56,57,59,65-67,72,73,75]$. Heart disease [55,57,59,72], mental illness $[59,67,71,75]$, brain damage $[70,72,74,75]$ and drinking and driving-related messages $[67,70,74,75]$ were used in four papers each $(15 \%)$, and pregnancy messages in three papers $(11 \%)[50,67,75]$. Four papers $(15 \%$; $[59,67,68,72])$, included other topics of messages such as diabetes, positive expectancies related to alcohol, warning against operating machinery or serving alcohol to minors. Fifteen papers (56\%) included images in the labels - ten of them included only graphic images (e.g., images of diseased body parts) $[55-57,61,62,64,65,68,69,76]$, two in- 
cluded both graphic and neutral images [66,75], one included only neutral images [67] and two included pictograms $[50,70]$.

In terms of label format, the majority of the papers formatted the labels to be clearly visible. This meant putting labels on the front of the pack, with a predetermined size and clear contrast between text and background and separation from the rest of the label (e.g., black text on white background with black or red border, such as $[55-57,61,63]$ or black text on a bright yellow background and red border, such as [51-54]). A complete description of the studied labels content and format is available in Supplementary Table S1.

\subsection{Key Label Characteristics - Impact on the Outcomes}

Results for all the studied outcomes from each of the papers are presented in Table 3. Below, the results are summarised based on the study aims identified through the two research questions: (1) studying overall label effectiveness by comparing image and text labels and (2) studying other label message characteristics and label formats.

\subsubsection{Label Effectiveness}

A large online UK study [63] did not find that adding a text warning to alcohol drink label changed perceived personal risk, motivation to drink less or perception of damaging drinking among adult drinkers. On the other hand, the Canadian real-world study, which included putting three types of labels (one of them a cancer warning) on the majority of alcohol drinks in the participating liquor stores in the studied region, found that these enhanced labels led to increased recall, cognitive processing and self-reported impact of drinking [51,54], as well as decreased alcohol sales [53] in the intervention municipality compared to the control municipality. While the whole intervention included both cancer warnings as well as low-risk guidelines/standard drink labels, it is not possible to completely disentangle the impact of solely the cancer warning labels from the results, although some results pointed to effects being driven predominantly by the cancer label. The one paper that looked specifically at the cancer labels [52] found that cancer labels increased knowledge of alcohol as a carcinogen, and that knowledge increased with time. Additionally, the authors found that knowledge about alcohol causing cancer was associated with a greater likelihood of supporting health warning labels.

\subsubsection{Label Content-Image vs. Text}

Among the eight papers that compared image and text labels, six had control group comparison $[55,61,62,64,67]$ and in two $[65,66]$, only text and image were compared. Six of the papers used graphic images (e.g., diseased body part); in one, the images were neutral, and one contained both graphic and neutral images.

In the studies where graphic images were used, comparison between text-only and image-and-text labels showed higher negative emotional arousal [55,56], fear $[55,56,61,65]$ and reactance [65] for the image-and-text labels among adults compared to text only labels, as well as lower product appeal $[64,65]$, label acceptability [55,56] and believability [65]. Moreover, in the study using neutral images [67], there were higher negative emotions for image-and-text labels compared to text-only were found among the secondary school students, but only for some messages. The same study also found that both types of labels are better than no label for increasing knowledge about alcohol-related risks when knowledge is low at baseline. While an online experiment showed a selection of alcoholic drinks in the given scenario to be lower after seeing image-and-text labels compared to text-only labels [55], this finding did not replicate in a naturalistic lab shopping setting [56] among adult regular drinkers, where none of the labels affected purchasing behaviour. In another study, both text-only and image-and-text labels decreased the speed of consumption compared to no label, with no significant difference between them [62]. 
Table 3. Results of the studies.

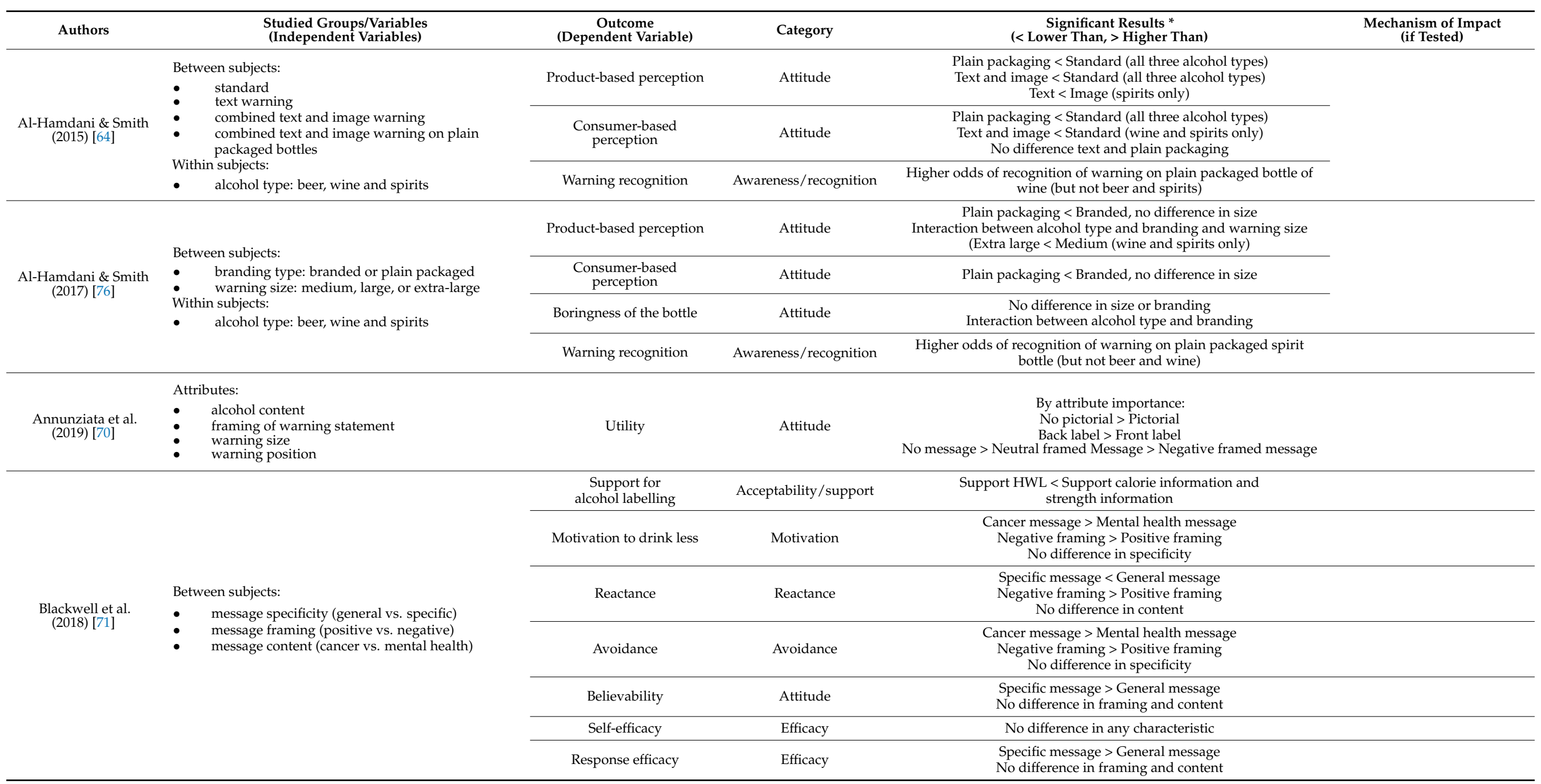


Table 3. Cont

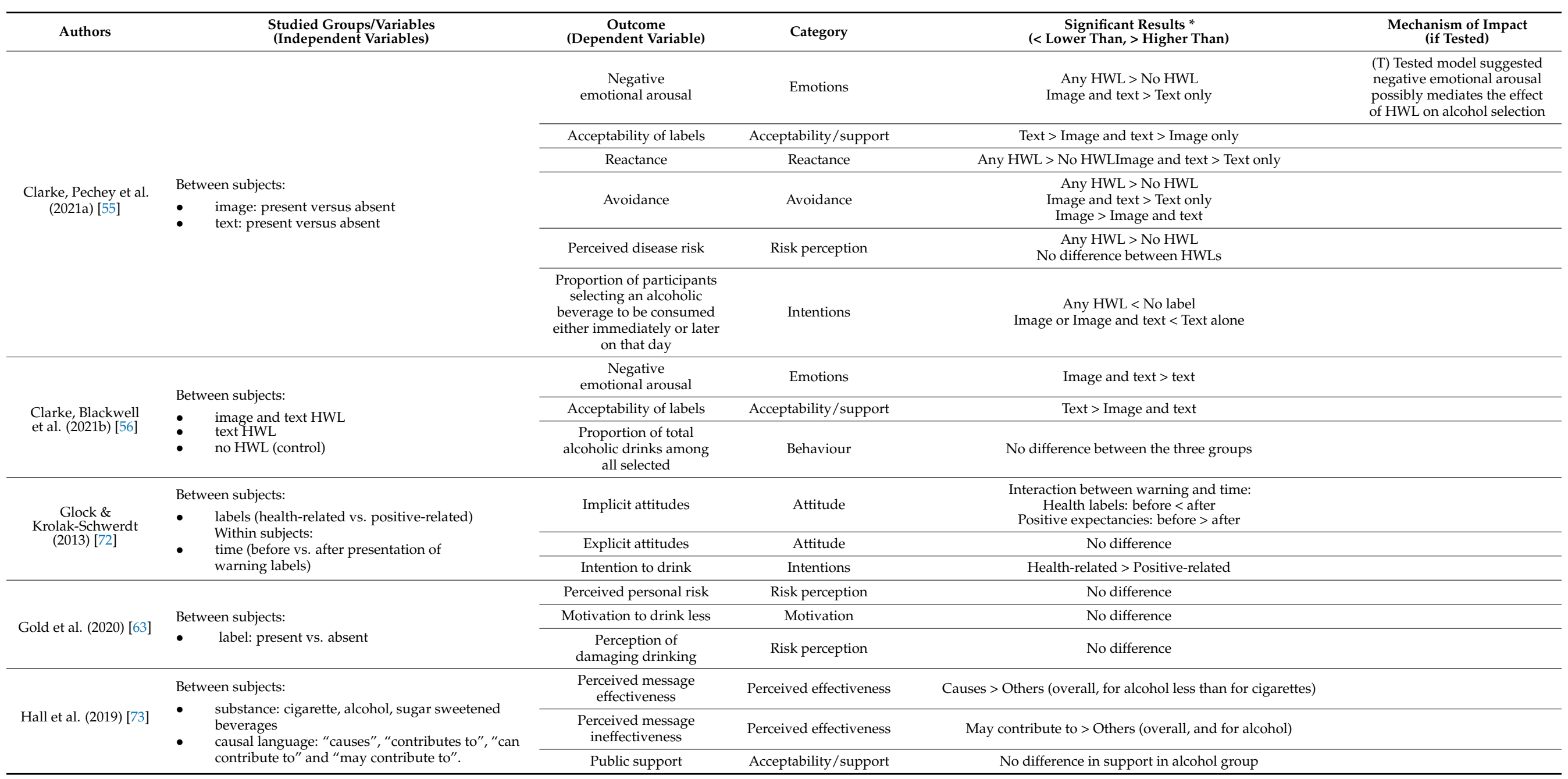


Table 3. Cont

\begin{tabular}{|c|c|c|c|c|c|}
\hline Authors & $\begin{array}{l}\text { Studied Groups/Variables } \\
\text { (Independent Variables) }\end{array}$ & $\begin{array}{c}\text { Outcome } \\
\text { (Dependent Variable) }\end{array}$ & Category & $\begin{array}{c}\text { Significant Results * } \\
\text { (< Lower Than, }>\text { Higher Than) }\end{array}$ & $\begin{array}{c}\text { Mechanism of Impact } \\
\text { (if Tested) }\end{array}$ \\
\hline \multirow{7}{*}{ Hall et al. (2020) [65] } & \multirow{7}{*}{$\begin{array}{l}\text { Between subjects: } \\
\bullet \quad \text { label: text vs. image and text } \\
\text { - efficacy information: present vs. absent } \\
\text { Within subjects: } \\
\text { - } \quad \text { substance: cigarette, alcohol, sugar sweetened } \\
\quad \text { beverages }\end{array}$} & $\begin{array}{l}\text { Perceived message } \\
\text { effectiveness }\end{array}$ & Perceived effectiveness & Image and text $>$ Text (overall, and for alcohol) & \\
\hline & & Believability & Attitude & Text > Image and text (overall, and for alcohol) & \\
\hline & & Fear & Emotions & Image and text $>$ Text (overall, and for alcohol) & \\
\hline & & Thinking about harms & Cognitive processing & Image and text $>$ Text (overall, and for alcohol) & \\
\hline & & Product appeal & Attitude & Text $>$ Image and text (overall, and for alcohol) & \\
\hline & & Policy support & Acceptability/support & Text > Image and text (overall, and for alcohol) & \\
\hline & & Self-efficacy & Efficacy & No difference in efficacy information presence & \\
\hline \multirow{3}{*}{$\begin{array}{l}\text { Hobin, } \\
\text { Schueri-Mychasiw } \\
\text { et al. (2020a) [51] }\end{array}$} & \multirow{3}{*}{$\begin{array}{l}\text { Between subjects: } \\
\text { - } \quad \text { labels: enhanced vs. regular }\end{array}$} & Recognition of labels & Awareness/recognition & Enhanced label > regular label & \\
\hline & & Cognitive processing & Cognitive processing & Enhanced label $>$ Regular label & $\begin{array}{l}\text { (T) Consumer attention to } \\
\text { and processing of label } \\
\text { messages partially mediated } \\
\text { the relationship between the } \\
\text { enhanced alcohol warning } \\
\text { labels and self-reported } \\
\text { drinking less } \rightarrow \text { suggests that } \\
\text { strengthening HWL will } \\
\text { increase their effectiveness } \\
\text { because they draw attention } \\
\text { to and increase processing of } \\
\text { the labels }\end{array}$ \\
\hline & & $\begin{array}{l}\text { Self-reported impact on } \\
\text { drinking (reduction) }\end{array}$ & Behaviour & Enhanced labels > Regular label & \\
\hline \multirow[b]{3}{*}{$\begin{array}{l}\text { Hobin, Weerasinghe } \\
\text { et al. (2020b) [52] }\end{array}$} & \multirow{3}{*}{$\begin{array}{l}\text { Between subjects: } \\
\text { - } \quad \text { labels: enhanced vs. regular }\end{array}$} & $\begin{array}{c}\text { Prompted and } \\
\text { unprompted recall }\end{array}$ & Awareness/recognition & $\begin{array}{c}\text { Enhanced label > Regular label (largest difference between } \\
\text { groups at T2) }\end{array}$ & \\
\hline & & $\begin{array}{l}\text { Knowledge of alcohol as } \\
\text { carcinogen }\end{array}$ & Knowledge & $\begin{array}{c}\text { Enhanced label > Regular label (largest difference between } \\
\text { groups at T3) }\end{array}$ & \\
\hline & & $\begin{array}{l}\text { Support for health } \\
\text { warning labels on alcohol } \\
\text { containers }\end{array}$ & Acceptability/support & $\begin{array}{c}\text { Intervention: Agree }(\text { Wave } 1=57.4 \% \text {; Wave } 2=57.3 \% \text {; } \\
\text { Wave } 3=61.3 \%) \\
\text { Comparison: Agree }(\text { Wave } 1=53.7 \% \text {; Wave } 2=51.6 \% \text {; } \\
\text { Wave } 3=53.7 \%)\end{array}$ & $\begin{array}{l}\text { (T) Knowledge about alcohol } \\
\text { causing cancer associated } \\
\text { with greater likelihood of } \\
\text { supporting health warning } \\
\text { labels in the study }\end{array}$ \\
\hline \multirow{3}{*}{$\begin{array}{l}\text { Hobin, Shokar et al. } \\
\text { (2020c) [54] }\end{array}$} & \multirow{3}{*}{$\begin{array}{l}\text { Between subjects: } \\
\text { - } \quad \text { labels: enhanced vs. regular }\end{array}$} & Unprompted recall & Awareness/recognition & Enhanced label > Regular label (cancer label) & \\
\hline & & Cognitive processing & Cognitive processing & Enhanced label $>$ Regular label & \\
\hline & & $\begin{array}{l}\text { Self-reported impact on } \\
\text { drinking (reduction) }\end{array}$ & Behaviour & Enhanced labels $>$ Regular label & \\
\hline $\begin{array}{l}\text { Jarvis \& Pettigrew } \\
\quad(2013)[74]\end{array}$ & $\begin{array}{l}\text { Attributes: } \\
\quad \text { brand } \\
\quad \text { alcohol content } \\
\quad \text { warning message (framed positive or negative) }\end{array}$ & Utility & Attitude & $\begin{array}{l}\text { Brand }>\text { Alcohol content }>\text { Warning statement } \\
\text { Two warning statements significant: one positive and one negative }\end{array}$ & \\
\hline
\end{tabular}


Table 3. Cont

\begin{tabular}{|c|c|c|c|c|c|}
\hline Authors & $\begin{array}{l}\text { Studied Groups/Variables } \\
\text { (Independent Variables) }\end{array}$ & $\begin{array}{c}\text { Outcome } \\
\text { (Dependent Variable) }\end{array}$ & Category & $\begin{array}{c}\text { Significant Results* } \\
\text { ( }<\text { Lower Than, }>\text { Higher Than) }\end{array}$ & $\begin{array}{c}\text { Mechanism of Impact } \\
\text { (if Tested) }\end{array}$ \\
\hline \multirow{3}{*}{$\begin{array}{l}\text { Jongenelis, Pettigrew } \\
\text { et al. (2018a) [58] }\end{array}$} & \multirow{3}{*}{$\begin{array}{l}\text { Between subjects: } \\
\text { - Source: single-source or multiple-source of } \\
\quad \text { message }\end{array}$} & $\begin{array}{l}\text { Intentions to reduce } \\
\text { alcohol consumption }\end{array}$ & Intentions & Multiple source $>$ Single source & \\
\hline & & Message believability & Attitude & Multiple source $>$ Single source & \\
\hline & & $\begin{array}{l}\text { Personal relevance of } \\
\text { the message }\end{array}$ & Attitude & Multiple source $>$ Single source & \\
\hline \multirow{2}{*}{$\begin{array}{l}\text { Jongenelis, Pratt et al. } \\
\text { (2018b) [59] }\end{array}$} & \multirow{2}{*}{$\begin{array}{l}\text { Between subjects: } \\
\text { - } \quad \text { Label content }\end{array}$} & $\begin{array}{c}\text { Alcohol as risk } \\
\text { factor beliefs }\end{array}$ & Knowledge & $\begin{array}{c}\text { Present message }>\text { Absent message (for all messages except for } \\
\text { liver damage) }\end{array}$ & \\
\hline & & $\begin{array}{l}\text { Intentions to reduce } \\
\text { alcohol consumption }\end{array}$ & Intentions & $\begin{array}{l}\text { Present message > Absent message (for cancer, diabetes and mental } \\
\text { illness message, but not for liver damage and heart disease) }\end{array}$ & \\
\hline \multirow{3}{*}{$\begin{array}{l}\text { Krischler \& Glock } \\
\text { (2015) [68] }\end{array}$} & \multirow{3}{*}{$\begin{array}{l}\text { Between subjects: } \\
\text { - labels (questions vs. statements vs. control) } \\
\text { Within subjects: } \\
\text { - } \quad \text { expectancy category (positive vs. negative, } \\
\quad \text { tension reduction vs. negative sedating vs. } \\
\text { socially related) }\end{array}$} & Drinking intentions & Intentions & No difference & \\
\hline & & $\begin{array}{c}\text { Individual outcome } \\
\text { expectancies }\end{array}$ & Attitude & No difference & \\
\hline & & $\begin{array}{c}\text { General outcome } \\
\text { expectancies }\end{array}$ & Attitude & No difference & \\
\hline \multirow{4}{*}{ Ma (2021) [69] } & \multirow{4}{*}{$\begin{array}{ll}\text { Between subjects: } \\
: \quad \text { narrative PWLs } \\
\bullet \quad \text { non-narrative PWLs } \\
\text { control (no stimulus) }\end{array}$} & $\begin{array}{l}\text { Worry about developing } \\
\text { alcohol-related cancer }\end{array}$ & Emotions & Narrative labels $>$ no labels & \\
\hline & & $\begin{array}{l}\text { Feelings of risk of } \\
\text { developing } \\
\text { alcohol-related cancer }\end{array}$ & Risk perception & Narrative labels $>$ no labels & \\
\hline & & $\begin{array}{l}\text { Perceived severity of harm } \\
\text { of developing } \\
\text { alcohol-related cancer }\end{array}$ & Risk perception & Narrative labels $>$ no labels & $\begin{array}{l}\text { (T) Mediation analysis found } \\
\text { that narrative PWLs (vs. } \\
\text { control) indirectly influenced } \\
\text { intentions through worry, bu } \\
\text { not through feelings of risk, } \\
\text { comparative likelihood, or } \\
\text { perceived severity. }\end{array}$ \\
\hline & & $\begin{array}{l}\text { Intentions to reduce } \\
\text { alcohol use }\end{array}$ & Intentions & No difference & \\
\hline $\begin{array}{l}\text { Monk et al. } \\
\text { (2017) [66] }\end{array}$ & $\begin{array}{l}\text { Between subjects: } \\
\quad \quad \text { image: graphic or neutral } \\
\quad \text { area of interest: text or image } \\
\text { positive expectancy change: increase or } \\
\text { decrease/no change }\end{array}$ & Dwell time & Attention & $\begin{array}{l}\text { Image }>\text { Text (overall and in the positive expectancies increase } \\
\text { group)No difference between graphic or neutral, and between } \\
\text { increase and decrease in positive expectancies }\end{array}$ & \\
\hline \multirow{3}{*}{$\begin{array}{l}\text { Morgenstern et al. } \\
\quad(2021)[67]\end{array}$} & \multirow{3}{*}{$\begin{array}{l}\text { Between subjects: } \\
\text { - } \quad \text { position of the HWL on the questionnaire (before } \\
\text { vs. after alcohol items) } \\
\text { type of HWL (text only vs. image and text) } \\
\text { - content of the HWL (one out of a pool of ten) }\end{array}$} & $\begin{array}{l}\text { Knowledge about } \\
\text { alcohol-related risks }\end{array}$ & Knowledge & Any label >No label (for cancer and liver cirrhosis) & \\
\hline & & Self-report of alcohol use & Behaviour & No difference & \\
\hline & & Negative emotions & Emotions & $\begin{array}{c}\text { Text and image > Text only (only for some messages-driving, liver } \\
\text { cirrhosis, pharmaceuticals, minors) }\end{array}$ & \\
\hline
\end{tabular}


Table 3. Cont

\begin{tabular}{|c|c|c|c|c|c|}
\hline Authors & $\begin{array}{l}\text { Studied Groups/Variables } \\
\text { (Independent Variables) }\end{array}$ & $\begin{array}{c}\text { Outcome } \\
\text { (Dependent Variable) }\end{array}$ & Category & $\begin{array}{c}\text { Significant Results } * \\
\text { (< Lower Than, }>\text { Higher Than) }\end{array}$ & $\begin{array}{l}\text { Mechanism of Impact } \\
\text { (if Tested) }\end{array}$ \\
\hline \multirow{3}{*}{$\begin{array}{l}\text { Pechey et al. } \\
\text { (2020) [57] }\end{array}$} & \multirow{3}{*}{$\begin{array}{l}\text { Between subjects: } \\
\text { - message type ( } 7 \text { messages of different health } \\
\text { - consequences) } \\
\text { image type ( } 3 \text { different graphic images per health } \\
\text { consequence) }\end{array}$} & $\begin{array}{c}\text { Negative } \\
\text { emotional arousal }\end{array}$ & Emotions & $\begin{array}{c}\text { Bowel cancer }>\text { Others }(>\text { liver cancer }>\text { liver cirrhosis }>\text { heart } \\
\text { disease }>\text { liver disease }>7 \text { types of cancer }>\text { breast cancer) }\end{array}$ & \\
\hline & & $\begin{array}{l}\text { Desire to consume the } \\
\text { labelled product }\end{array}$ & Motivation & $\begin{array}{c}\text { Bowel cancer }<\text { Liver cirrhosis }<\text { Breast cancer }<\text { Liver cancer }< \\
\text { Heart disease }<\text { Liver disease }<7 \text { types of cancer }\end{array}$ & \\
\hline & & Acceptability of the label & Acceptability/support & $\begin{array}{c}\text { Low overall acceptability, Bowel cancer }<\text { Others (Breast cancer }< \\
\text { Liver disease }<7 \text { types of cancer }<\text { Liver cancer }<\text { Liver disease }< \\
\text { Liver cirrhosis) }\end{array}$ & \\
\hline \multirow{3}{*}{$\begin{array}{l}\text { Pettigrew et al. } \\
\text { (2016) [60] }\end{array}$} & \multirow{3}{*}{$\begin{array}{l}\text { Between subjects: } \\
\text { - content of the message ( } 6 \text { messages) } \\
\text { Within subjects: } \\
\text { - before and after (only for intention) }\end{array}$} & $\begin{array}{l}\text { Intention to reduce } \\
\text { alcohol consumption }\end{array}$ & Intentions & $\begin{array}{c}\text { Pre }<\text { Post } \\
\text { No difference between messages }\end{array}$ & \\
\hline & & $\begin{array}{l}\text { Convincingness of } \\
\text { the message }\end{array}$ & Attitude & Bowel cancer $>$ Other messages & \\
\hline & & $\begin{array}{l}\text { Personal relevance of } \\
\text { the message }\end{array}$ & Attitude & Bowel cancer > Other messages & \\
\hline \multirow[b]{2}{*}{$\begin{array}{l}\text { Pham et al. } \\
\text { (2018) [50] }\end{array}$} & \multirow{2}{*}{$\begin{array}{l}\text { Between subjects: } \\
\text { - } \quad \text { colour change (use of red colouring instead of } \\
\text { black) } \\
\text { - } \quad \text { size of the HWL (increase by } 50 \% \text { ) } \\
\text { changes in both colour and size } \\
\text { - control (existing label) }\end{array}$} & Attention & Attention & Enhanced colour and size $>$ Other groups & \\
\hline & & $\begin{array}{l}\text { Number } \\
\text { and } \\
\text { length of } \\
\text { visual } \\
\text { fixations }\end{array}$ & Attention & No difference between groups & \\
\hline \multirow{5}{*}{$\begin{array}{l}\text { Sillero-Rejon et al. } \\
\quad \text { (2018) [75] }\end{array}$} & \multirow{5}{*}{$\begin{array}{l}\text { Between subjects: } \\
\text { • } \quad \text { self-affirmation (self-affirmed vs. control) } \\
\text { Within-subject: } \\
\text { - } \quad \text { warning severity (moderately-severe vs. } \\
\quad \text { highly-severe) }\end{array}$} & Visual attention & Attention & No difference in severity or self-affirmation & \\
\hline & & Reactance & Reactance & $\begin{array}{l}\text { Highly severe > Moderately severe } \\
\text { No difference in self-affirmation }\end{array}$ & \\
\hline & & $\begin{array}{l}\text { Perceived susceptibility to } \\
\text { health risks }\end{array}$ & Risk perception & No difference in severity or self-affirmation & \\
\hline & & $\begin{array}{l}\text { Perceived warning } \\
\text { effectiveness }\end{array}$ & Perceived effectiveness & $\begin{array}{l}\text { Highly severe > Moderately severe } \\
\text { No difference in self-affirmation }\end{array}$ & \\
\hline & & Motivation to drink less & Motivation & $\begin{array}{l}\text { Highly severe }>\text { Moderate severe } \\
\text { No difference in self-affirmation }\end{array}$ & \\
\hline \multirow[t]{2}{*}{$\begin{array}{l}\text { Stafford \& Salmon } \\
\quad(2017)[62]\end{array}$} & \multirow{2}{*}{$\begin{array}{l}\text { Between subjects: } \\
\text { - } \quad \text { no HWL } \\
\text { - } \quad \text { image and text HWL }\end{array}$} & Product design evaluation & Attitude & $\begin{array}{l}\text { Image and text } \mathrm{HWL}<\mathrm{No} H W L \\
\text { No difference between text only and no HWL }\end{array}$ & $\begin{array}{l}\text { (H) The mechanism } \\
\text { responsible for slower } \\
\text { consumption is theorised to } \\
\text { be due to higher levels of fear } \\
\text { arousal in the two health } \\
\text { warning conditions }\end{array}$ \\
\hline & & $\begin{array}{l}\text { The duration to consume } \\
\text { the test beverage }\end{array}$ & Behaviour & $\begin{array}{c}\text { No HWL }<\text { Text HWL } \\
\text { No HWL }<\text { Image and text HWL } \\
\text { No difference between text HWL and image and text HWL }\end{array}$ & \\
\hline
\end{tabular}


Table 3. Cont

\begin{tabular}{|c|c|c|c|c|c|}
\hline Authors & $\begin{array}{l}\text { Studied Groups/Variables } \\
\text { (Independent Variables) }\end{array}$ & $\begin{array}{c}\text { Outcome } \\
\text { (Dependent Variable) }\end{array}$ & Category & $\begin{array}{c}\text { Significant Results } * \\
(<\text { Lower Than, }>\text { Higher Than) }\end{array}$ & $\begin{array}{c}\begin{array}{c}\text { Mechanism of Impact } \\
\text { (if Tested) }\end{array} \\
\end{array}$ \\
\hline \multirow{3}{*}{$\begin{array}{l}\text { Wigg \& Stafford } \\
\text { (2016) [61] }\end{array}$} & \multirow{3}{*}{\begin{tabular}{l}
\multicolumn{2}{l}{ Between subjects: } \\
$\bullet \quad$ no HWL \\
$\bullet \quad$ text only HWL \\
image and text HWL
\end{tabular}} & Fear arousal & Emotions & $\begin{array}{c}\text { Image and text HWL }>\text { Text HWL } \\
\text { Image and text HWL }>\text { No HWL } \\
\text { No difference between text HWL and no HWL }\end{array}$ & \\
\hline & & Risk perception & Risk perception & $\begin{array}{c}\text { Image and text HWL }>\text { No HWL } \\
\text { No difference between text HWL and no HWL } \\
\text { No difference between image and text HWL and text HWL }\end{array}$ & \\
\hline & & $\begin{array}{l}\text { Intention to quit/reduce } \\
\text { alcohol consumption }\end{array}$ & Intentions & $\begin{array}{l}\text { Image and text HWL }>\text { No HWL } \\
\text { No difference between text HWL and no HWL } \\
\text { No difference between image and text HWL and text HWL }\end{array}$ & \\
\hline
\end{tabular}

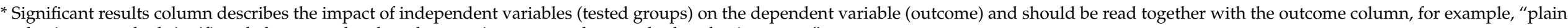
packaging group had significantly lower product based perception compared to standard packaging group". 
The two studies actively looking at the differences between graphic and neutral images did not find any difference between the two in attention [66,75], although the latter found higher avoidance, reactance, perceived warning effectiveness and motivation to drink less in graphic compared to neutral images.

\subsubsection{Label Content-Message Characteristics}

Several papers also compared different messages or their characteristics. In terms of message content, in Australia, one study among adult drinkers found increased intention to reduce drinking for cancer, diabetes and mental illness messages, but not for heart disease and liver damage-related messages [59]. Another study found that a bowel cancer message had the highest believability, convincingness and personal relevance [60]. A bowel cancer message also had the highest negative emotional arousal, lowest desire to consume the labelled product and lowest acceptability in a large-scale UK online study of adult drinkers [57], but in this study, all the labels included graphic photos, which might have influenced the outcome. Another large scale online experiment in the UK among adult drinkers [71] compared a cancer message to a mental health message and found the former led to a higher motivation to drink less and higher avoidance, but there was no difference in reactance, believability, self-efficacy and response efficacy. Another smaller study found that positive expectancy-related messages lowered drinking intentions compared to health-related messages among students from Germany and Luxembourg [72]. In a German sample of secondary school students, cancer and liver cirrhosis messages, but not a pregnancy label, increased knowledge about alcohol-related risks [67].

In terms of framing, the discrete choice experiments gave mixed results $[70,74]$; in the first study, no message at all was more preferred than any message in a sample of Italian and French young wine drinkers, and in the latter, the negative message was less preferred than the neutral message among Australian students. In an online study of British adult drinkers [71], a negative framing led to a higher motivation to drink less, with increased reactance and avoidance compared to a positive framing, but did not influence believability, self-efficacy and response efficacy. This study also looked at specificity and found that specific messages led to lower reactance, higher response efficacy and higher believability compared to general messages, but there was no difference in motivation to drink less, avoidance and self-efficacy.

One study looked at whether framing labels as questions or statements impacts attitude and intentions, and found no difference [68]. Another study compared narrative and non-narrative labels and found narrative labels led to higher worry about developing alcohol-related cancer and higher feelings of risk of developing alcohol-related cancer, but no difference in intentions to reduce alcohol use [69]. The study examining causal language [73] found that using the word "causes" had higher perceived message effectiveness, and "may contribute to" had the highest perceived ineffectiveness, but there was no difference in support for any of the labels. Finally, a study of Australian adult drinkers found that receiving the message from multiple sources is better than receiving a message from one single source [58].

\subsubsection{Label Format}

Finally, four studies examined label format: branding/plain packaging [64,76], warning size $[50,70,76]$ and warning position [70]. Two smaller-scale Canadian studies found that plain packaging (removing any branding) decreased product-based and consumerbased perception [64,76]. The latter study [76] also found that after a certain size of the warning (50\% of the bottle), there is no further increased difference in outcomes. In a discrete choice experiment, young Italian and French wine drinkers preferred to have warning sizes as little as possible, and on the back of the label rather than on the front of the label [70]. Finally, one study [50] found that an increase in size and change in prominent colour was effective in self-reported measures of attention among the Australian drinkers, but not when measured directly with eye-tracking. 


\section{Discussion}

This review aimed to examine the scope of the (quasi-)experimental research on novel and enhanced alcohol health warning labels and to identify the key characteristics of the effective warning labels. Twenty-seven papers were included, the majority of which were published in 2018 or later, indicating an uptake in interest surrounding the topic from the research community (recognised also in other broader reviews [45]). This is aligned with the increase in policy-related labelling discussions, such as in Codex Alimentarius, at the EU or national level (e.g., [77,78]). All of the research has been undertaken in Western countries, where alcohol labelling has either not been updated since its implementation decades ago (e.g., Canada, US), carried out on a voluntary basis (e.g., Australia, UK) or not mandated at all (e.g., many of the EU countries). Given high alcohol consumption in those regions [79], this may reflect the recognition of labelling of potentially effective means of reducing alcohol-related harm-although it is debatable if this focus adds to or replaces focus on "best buys" policies [80], such as taxation, pricing and advertising restrictions. In addition, the majority of the studies focused on general population, which reflects a shift from findings of previous review [43], where less than a third of studies were targeting the broader population, and the remaining were targeting younger populations and (pregnant) women.

In terms of content, most commonly, the studied messages were referring to cancer$70 \%$ of papers included at least one cancer-related message, out of which the most commonly mentioned cancers were bowel, breast and liver cancer. This was followed by liver cirrhosis or liver disease messages, used in a third of papers. Other topics of messages were heart disease, mental illness, brain damage, pregnancy, drinking and driving and other (e.g., positive expectancies surrounding alcohol). This brings us to the question on how to select the content of the message to be included on the labels. In the papers included in this review, researchers have commonly either developed messages based on "evidence on alcohol-related harm" or have taken messages used in other studies. We would argue that the message first has to reflect evidence-based risk (e.g., that causal relationship between alcohol and the outcome has been clearly demonstrated [3]), and then the message should be tailored to the context in which the labels will be displayed, based on the burden of disease. Another factor that has been found to be important for the selection of the message is also its novelty for the given audience [16,53]. For example, in the EU, $29.4 \%$ of all alcohol-attributable deaths and $19.4 \%$ of all attributable DALYs are due to cancers [81], while awareness of the risk between alcohol and cancer is low [15]. Based on these guidelines we can conclude that cancer warnings should be a priority when mandating health warning labels in this region.

Next, the focus of research implies the researcher's theory of change-the mechanisms by which they consider labelling to work [82] —and this will influence the targeted outcomes and how effectiveness is measured. This is relevant because the policy debate will inevitably discuss the merit of the health warning labels based on their "effectiveness" previously, one of the arguments in the World Trade Organisation's discussions was that labels are considered unnecessary interference with international trade because there is no scientific evidence to support such warnings as an effective public health measure [83]. Based on the scope of the existing research, we propose that two possible theories of how labelling brings about change are currently being investigated. The first one is focused on persuasion and short-term individual behaviour change, and optimises using health warning labels that aim to elicit reactance, avoidance, fear and other negative emotional reactions as means of changing behaviour, drawing heavily on the experience from tobacco warning labels. For this line of research, there is no real-world evidence from the alcohol field, and mixed evidence from lab studies regarding (short-term) behaviour [56,62]. The (online and lab) studies that focused on studying the graphic images on labels found that they elicited higher negative emotional arousal, fear and reactance, and were not well accepted by the participants. These results are also corroborated by the metanalysis investigating the impact of HWL in both alcohol and food products, and found that while health warning labels reduce product selection compared to no labels, the difference between 
image-and-text HWLs and text-only HWLs was not statistically significant (although in the former, the effect was slightly larger) [44].

The second theory of change sees labelling as a tool to raise awareness of alcoholrelated risks as a part of a comprehensive strategy to reduce alcohol-related harm (which should include other alcohol policies and interventions), and emphasises optimising labels to increase attention to and cognitive processing of the information on the labels, leading to increased knowledge (e.g., on alcohol health risks). This approach is more in line with system perspectives [84] and its focus is to use health warning labels to arrive at a better informed population that could in turn lead to more support for other alcohol policies. Individual short-term behaviour change might be a welcome by-product of this approach, but in the long term, labels can be seen as a tool to help with changing societal conversation and norms surrounding alcohol (as suggested also by O'Brien [85]). In terms of evidence, the best current evidence points to the second theory of change as having greater potential to be explored in future research and practice. The most comprehensive study to date and the only one measuring long-term effects in the real world [86] showed that simple, specific and well-visible messages led to an increase in knowledge (with associated support for stronger alcohol policies [87]) and change in the amount of alcohol consumed, partially mediated by the consumer attention to and processing of label messages [54]. The labels were also well accepted and supported by the population, as well as the drinkers [51].

It is important to note that this study [86] was implemented in context with existing relatively strict other policy measures-e.g., national monopoly on the alcohol stores (alcohol being sold separately in the government-owned liquor stores), and enhanced label replacing existing label (rather than no label). Nevertheless, a key approach to label development and implementation in this study is a good practice example and should be used in any future attempts to study alcohol HWLs in different contexts. This means developing labels in consultation with local stakeholders, including a range of different messages (not only health but also related to standard drinks and lower-risk guidelines) and including evidence-based messages for which there was low awareness at baseline and are relevant to the population. This study [83] also points to the unintended consequences that can be anticipated-originally, the study was planned to run for eight months with all three labels, but the alcohol industry intervened and threatened with a lawsuit if cancer warning labels were to continue being applied on the products [83]. Thus, cancer labels were only applied to the alcohol products for one instead of eight months. This shows that in any attempts to mandate evidence-based alcohol health warning labels, strong opposition from the industry can be expected, similar to what happened in the tobacco field [88].

Thus, while there should be some lessons from tobacco taken for alcohol health warning labels, we must be careful to still consider alcohol in its own context, and note that the evolution of alcohol legislation lags behind the tobacco field by a couple of decades. There is evidence for the effectiveness of pictorial labels for tobacco [36], but even here, the argument can be made that labels have been part of a broader approach that involved international collaboration and changed societal norms around tobacco [89], and their purpose was perhaps less to deter smokers from smoking than to indirectly prevent younger populations from taking up smoking, as tobacco warning labels replaced packaging as the last venue of marketing after other forms of advertising had been prohibited [38]. The value of adding images on alcohol labels might be to help with attracting attention and take away space from the branding of the product, but not enough studies with only neutral images or symbols have been conducted to make any firm conclusions.

The main implications for research and policy based on the results of this review are summarised in Table 4. We would like to note that the same theory of change useful for health warnings could also be useful to develop and test for optimal nutrition information on alcohol labels (not direct focus on changing behaviour, but focus on providing the information), although as previously mentioned, the issue with nutritional labels is to first align requirements for alcoholic beverages with existing labelling requirements for food and non-alcoholic beverages. 
Table 4. Research and policy implications.

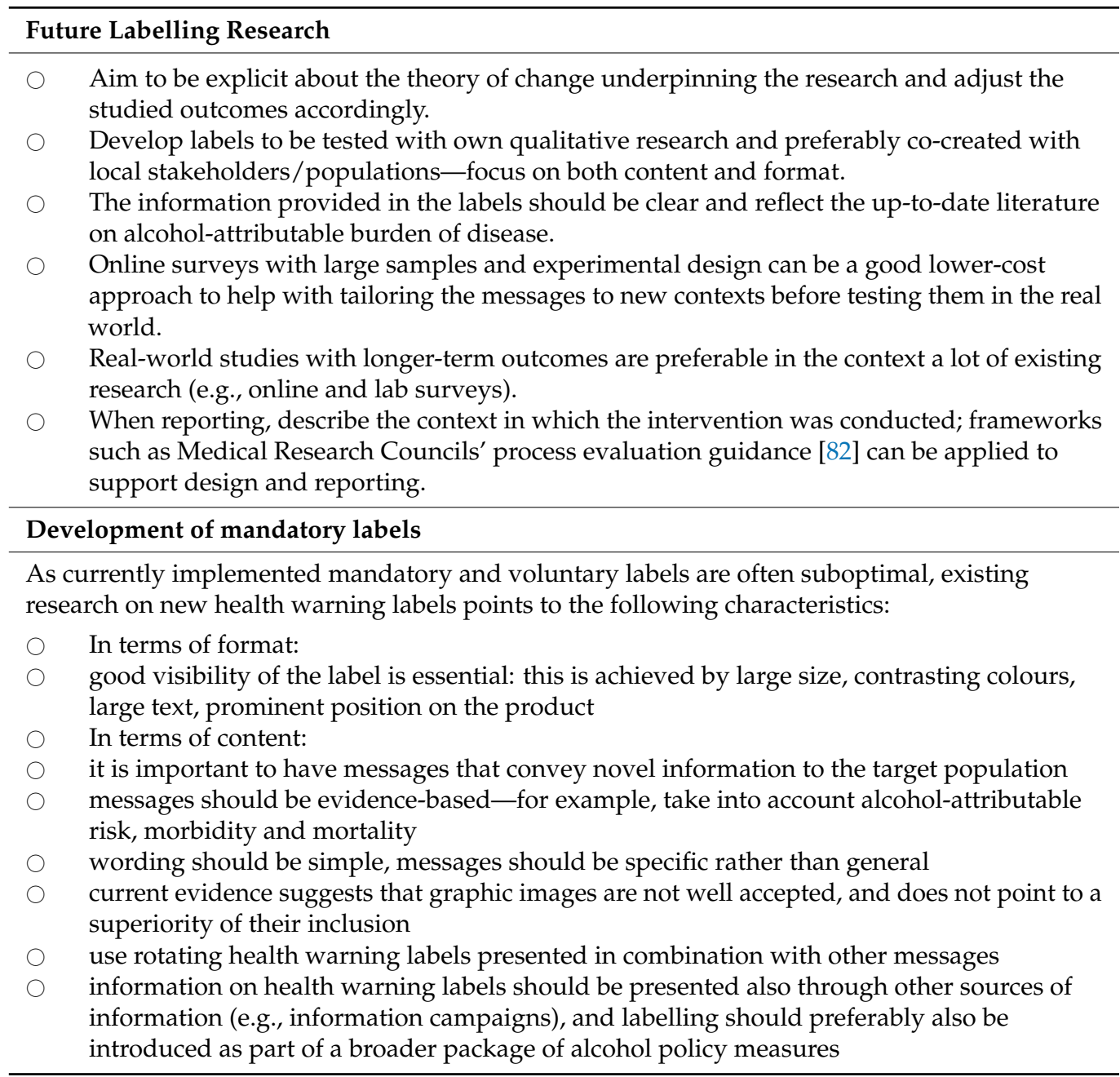

\subsection{Limitations}

Our review is subject to general limitations of scoping reviews: we did not appraise studies for quality, and the conclusions are still somewhat broad and qualitative. In terms of this specific review, we narrowed the scope of our investigation to health warning labels and overall outcomes and we did not focus on other labels, such as lower-risk drinking guidelines or standard drinks, even though some research suggests it might be worth taking them into account simultaneously. We also did not investigate differential impact of labels on different subgroups. Evidence from Canada points to some subgroup differences in cognitive processing of the labels, although concludes that vulnerable populations still attended to the labels [52]. Our search was also focused only on peer-reviewed English language papers; therefore, we might have missed peer-reviewed papers in other languages and non-peer-reviewed research.

\subsection{Conclusions}

The majority of the current evidence regarding alcohol health warning labels comes from very short interventions in the online or the laboratory setting as opposed to the real world, and there is rather large diversity in the content and format of labels being evaluated. Nevertheless, evidence from real-world, long-term interventions shows that alcohol health warning labels designed to be visible and contain novel and specific information have the potential to be part of an effective labelling strategy. Labelling should be seen as tool to raise awareness and a small part of wider alcohol prevention and policy approach. Future research could focus on importance of labels in a broader societal context, and go beyond 
individual behaviour change, recognising that change in social norms can be a complex, multifaceted and long-term process.

Supplementary Materials: The following are available online at https://www.mdpi.com/article/10 $.3390 /$ nu13093065/s1, Table S1. Label development, content and format.

Author Contributions: Conceptualisation: D.K., P.A., E.J.-L.; Methodology: D.K., P.A., E.J.-L.; Investigation: D.K.; Data analysis: D.K., Supervision; E.J.-L.; Writing—original draft: D.K.; WritingReview and Editing: D.K., P.A., E.J.-L. All authors have read and agreed to the published version of the manuscript.

Funding: Part of this research was produced under the service contract AlHaMBRA Project (Alcohol Harm-Measuring and Building Capacity for Policy Response and Action, Contract No. 20197105).

Institutional Review Board Statement: Not applicable.

Informed Consent Statement: Not applicable.

Data Availability Statement: Extracted data are presented in the main and supplementary tables.

Acknowledgments: Part of this research was produced under the service contract AlHaMBRA Project (Alcohol Harm-Measuring and Building Capacity for Policy Response and Action, Contract No. 20197105). The information and views presented are those of the authors, and hence represent their sole responsibility. Accordingly, the information and views presented cannot be considered to reflect the views of all AlHaMBRA Project consortium members, the European Commission and/or the Health and Digital Executive Agency or any other body of the European Union. The European Commission and the Agency do not accept any responsibility for use that may be made of the information contained herein.

Conflicts of Interest: The authors declare no conflict of interest.

\section{Appendix A}
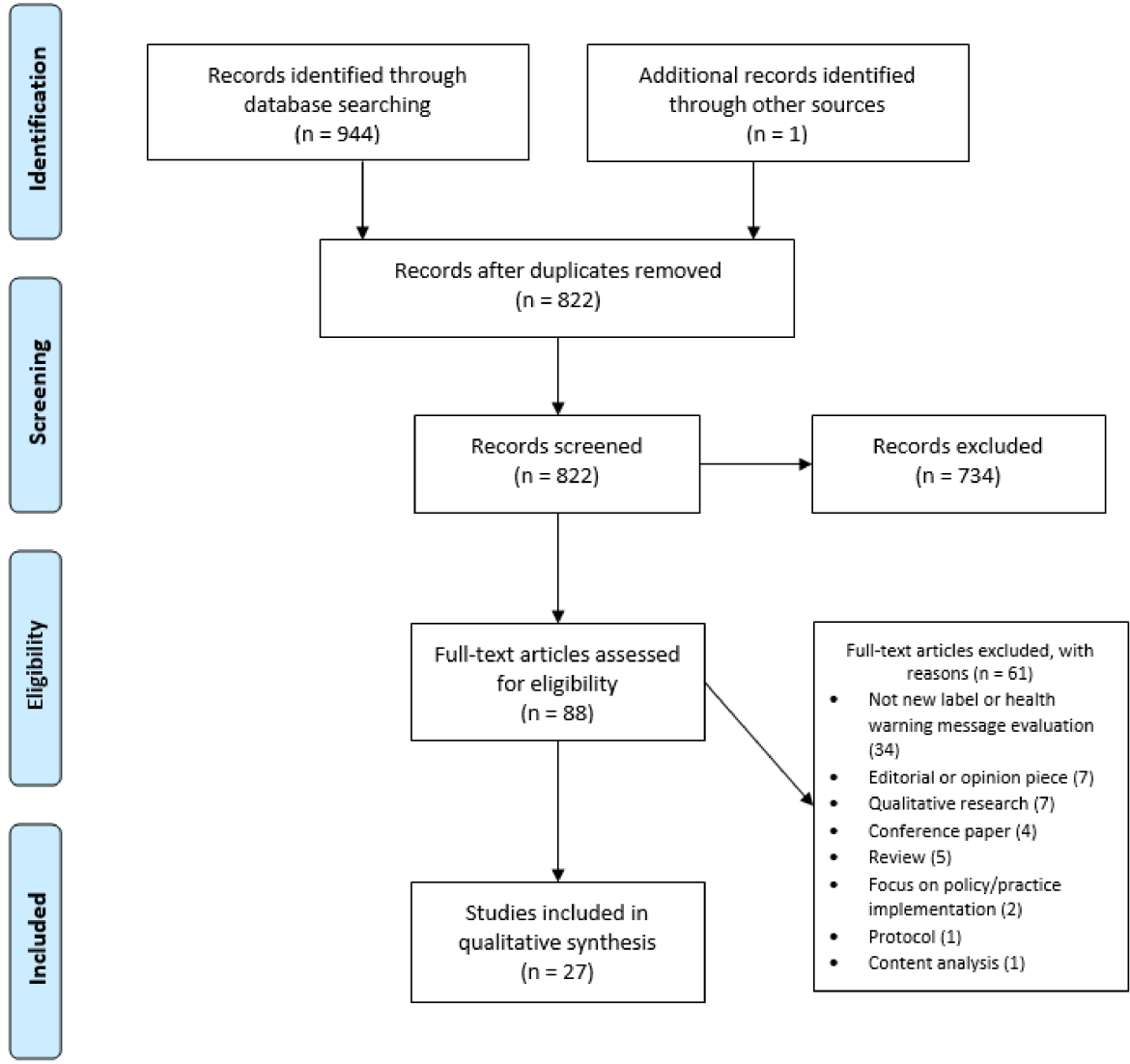

Figure A1. PRISMA Diagram. 


\section{References}

1. Shield, K.; Manthey, J.; Rylett, M.; Probst, C.; Wettlaufer, A.; Parry, C.D.H.; Rehm, J. National, regional, and global burdens of disease from 2000 to 2016 attributable to alcohol use: A comparative risk assessment study. Lancet Public Health 2020, 5, e51-e61. [CrossRef]

2. Okaru, A.O.; Lachenmeier, D.W. Margin of exposure analyses and overall toxic effects of alcohol with special consideration of carcinogenicity. Nutrients 2021, in press.

3. Rehm, J.; Gmel Sr, G.E.; Gmel, G.; Hasan, O.S.M.; Imtiaz, S.; Popova, S.; Probst, C.; Roerecke, M.; Room, R.; Samokhvalov, A.V.; et al. The relationship between different dimensions of alcohol use and the burden of disease-An update. Addiction 2017, 112, 968-1001. [CrossRef]

4. Rehm, J.; Taylor, B.; Mohapatra, S.; Irving, H.; Baliunas, D.; Patra, J.; Roerecke, M. Alcohol as a risk factor for liver cirrhosis: A systematic review and meta-analysis. Drug Alcohol Rev. 2010, 29, 437-445. [CrossRef]

5. Bagnardi, V.; Rota, M.; Botteri, E.; Tramacere, I.; Islami, F.; Fedirko, V.; Scotti, L.; Jenab, M.; Turati, F.; Pasquali, E.; et al. Alcohol consumption and site-specific cancer risk: A comprehensive dose-response meta-analysis. Br. J. Cancer 2015, 112, 580-593. [CrossRef] [PubMed]

6. International Agency for Research on Cancer IARC. IARC Monographs on the Evaluation of Carcinogenic Risks to Humans: Volume 96: Alcohol Consumption and Ethyl Carbamate; International Agency for Research on Cancer IARC: Lion, France, 2010 ; Volume 96.

7. International Agency for Research on Cancer. Monographs on the Evaluation of Carcinogenic Risks to Humans: Alcohol Drinking; International Agency for Research on Cancer IARC: Lion, France, 1988; Volume 44.

8. Grant, B.F.; Goldstein, R.; Saha, T.D.; Chou, S.P.; Jung, J.; Zhang, H.; Pickering, R.P.; Ruan, W.J.; Smith, S.M.; Huang, B.; et al. Epidemiology of DSM-5 Alcohol Use Disorder results from the national epidemiologic survey on alcohol and related conditions III. JAMA Psychiatry 2015, 72, 757-766. [CrossRef] [PubMed]

9. Rehm, J.; Roerecke, M. Cardiovascular effects of alcohol consumption. Trends Cardiovasc. Med. 2017, 27, 534-538. [CrossRef]

10. Imtiaz, S.; Shield, K.D.; Roerecke, M.; Samokhvalov, A.V.; Lonnroth, K.; Rehm, J. Alcohol consumption as a risk factor for tuberculosis: Meta-analyses and burden of disease. Eur. Respir. J. 2017, 50, 1700216. [CrossRef]

11. Bellos, S.; Skapinakis, P.; Rai, D.; Zitko, P.; Araya, R.; Lewis, G.; Lionis, C.; Mavreas, V. Longitudinal association between different levels of alcohol consumption and a new onset of depression and generalized anxiety disorder: Results from an international study in primary care. Psychiatry Res. 2016, 243, 30-34. [CrossRef]

12. Boden, J.M.; Fergusson, D.M. Alcohol and depression. Addiction 2011, 106, 906-914. [CrossRef]

13. Popova, S.; Lange, S.; Shield, K.; Mihic, A.; Chudley, A.E.; Mukherjee, R.A.S.; Bekmuradov, D.; Rehm, J. Comorbidity of fetal alcohol spectrum disorder: A systematic review and meta-analysis. Lancet 2016, 387, 978-987. [CrossRef]

14. Testa, M.; Quigley, B.M.; Eiden, R. The Effects of Prenatal Alcohol Exposure on Infant Mental Development: A Meta-Analytical Review. Alcohol Alcohol. 2003, 38, 295-304. [CrossRef]

15. Scheideler, J.K.; Klein, W.M.P. Awareness of the Link between Alcohol Consumption and Cancer across the World: A Review. Cancer Epidemiol. Biomark. Prev. 2018, 27, 429-437. [CrossRef]

16. Vallance, K.; Stockwell, T.; Zhao, J.; Shokar, S.; Schoueri-Mychasiw, N.; Hammond, D.; Greenfield, T.K.; McGavock, J.; Weerasinghe, A.; Hobin, E. Baseline Assessment of Alcohol-Related Knowledge of and Support for Alcohol Warning Labels Among Alcohol Consumers in Northern Canada and Associations With Key Sociodemographic Characteristics. J. Stud. Alcohol Drugs 2020, 81, 238-248. [CrossRef] [PubMed]

17. Calvert, C.M.; Toomey, T.; Jones-Webb, R. Are people aware of the link between alcohol and different types of Cancer? BMC Public Health 2021, 21, 1-10. [CrossRef] [PubMed]

18. Thomsen, K.L.; Christensen, A.S.P.; Meyer, M.K.H. Awareness of alcohol as a risk factor for cancer: A population-based cross-sectional study among 3000 Danish men and women. Prev. Med. Rep. 2020, 19, 101156. [CrossRef]

19. Martin-Moreno, J.M.; Harris, M.E.; Breda, J.; Møller, L.; Alfonso-Sanchez, J.L.; Gorgojo, L. Enhanced labelling on alcoholic drinks: Reviewing the evidence to guide alcohol policy. Eur. J. Public Health 2013, 23, 1082-1087. [CrossRef] [PubMed]

20. World Health Organisation Global Information System on Alcohol and Health (GISAH). Available online: http:/ / apps.who.int/ gho/data/node.gisah.A1191?lang=en\&showonly=GISAH (accessed on 27 August 2021).

21. Government of Canada Labelling Requirements for Alcoholic Beverages. Available online: https://inspection.canada.ca/foodlabel-requirements/labelling/industry/alcohol/eng/1392909001375/1392909133296 (accessed on 11 June 2021).

22. European Commission Regulation (EU) No 1169/2011 of the European Parliament and of the Council of 25 October 2011 on the Provision of Food Information to Consumers. Available online: https:/ / eur-lex.europa.eu/legal-content/EN/ALL/?uri= CELEX\%3A32011R1169 (accessed on 27 August 2021).

23. Food Standards Australia and New Zealand Labelling of Alcoholic Beverages. Available online: https: / /www.foodstandards. gov.au/consumer/labelling/Pages/Labelling-of-alcoholic-beverages.aspx (accessed on 11 June 2021).

24. Alcohol and Tobacco Tax and Trade Bureau Labeling Resources. Available online: https://www.ttb.gov/labeling/labelingresources (accessed on 11 June 2021).

25. Maynard, O.M.; Langfield, T.; Attwood, A.S.; Allen, E.; Drew, I.; Votier, A.; Munafò, M.R. No Impact of Calorie or Unit Information on Ad Libitum Alcohol Consumption (Special Issue: Communicating messages about drinking). Alcohol Alcohol. 2018, 53, 12-19. [CrossRef] [PubMed] 
26. Maynard, O.; Blackwell, A.; Munafò, M.; Attwood, A. Know Your Limits: Labelling Interventions to Reduce Alcohol Consumption; Alcohol Research UK: London, UK, 2018.

27. Croker, H.; Packer, J.; Russell, S.J.; Stansfield, C.; Viner, R.M. Front of pack nutritional labelling schemes: A systematic review and meta-analysis of recent evidence relating to objectively measured consumption and purchasing. J. Hum. Nutr. Diet. 2020, 33, 518-537. [CrossRef]

28. Kelly, B.; Jewell, J. What Is the Evidence on the Policy Specifications, Development Processes and Effectiveness of Existing Front-of-Pack Food Labelling Policies in the WHO European Region? WHO Regional Office for Europe: Copenhagen, Denmark, 2018.

29. Wilkinson, C.; Allsop, S.; Cail, D.; Chikritzhs, T.; Daube, M.; Kirby, G.; Mattick, R. Alcohol Warning Labels: Evidence of Effectiveness on Risky Alcohol Consumption and Short Term Outcomes; National Drug Research Institute: Bentley, WA, Australia, 2009.

30. Wilkinson, C.; Room, R. Warnings on alcohol containers and advertisements: International experience and evidence on effects. Drug Alcohol Rev. 2009, 28, 426-435. [CrossRef]

31. Dossou, G.; Gallopel-Morvan, K.; Diouf, J.-F. The effectiveness of current French health warnings displayed on alcohol advertisements and alcoholic beverages. Eur. J. Public Health 2017, 27, 699-704. [CrossRef]

32. Dumas, A.; Toutain, S.; Hill, C.; Simmat-Durand, L. Warning about drinking during pregnancy: Lessons from the French experience. Reprod. Health 2018, 15, 20. [CrossRef] [PubMed]

33. Coomber, K.; Martino, F.; Barbour, I.R.; Mayshak, R.; Miller, P.G. Do consumers “Get the facts"? A survey of alcohol warning label recognition in Australia. BMC Public Health 2015, 15, 816. [CrossRef] [PubMed]

34. Critchlow, N.; Jones, D.; Moodie, C.; Mackintosh, A.M.; Fitzgerald, N.; Hooper, L.; Thomas, C.; Vohra, J. Awareness of productrelated information, health messages and warnings on alcohol packaging among adolescents: A cross-sectional survey in the United Kingdom. J. Public Health 2020, 42, e223-e230. [CrossRef] [PubMed]

35. Kersbergen, I.; Field, M. Alcohol consumers' attention to warning labels and brand information on alcohol packaging: Findings from cross-sectional and experimental studies. BMC Public Health 2017, 17, 1-11. [CrossRef] [PubMed]

36. Noar, S.M.; Francis, D.B.; Bridges, C.; Sontag, J.M.; Ribisl, K.M.; Brewer, N.T. The impact of strengthening cigarette pack warnings: Systematic review of longitudinal observational studies. Soc. Sci. Med. 2016, 164, 118-129. [CrossRef]

37. Noar, S.M.; Francis, D.B.; Bridges, C.; Sontag, J.M.; Brewer, N.T.; Ribisl, K.M. Effects of Strengthening Cigarette Pack Warnings on Attention and Message Processing: A Systematic Review. J. Mass Commun. Q. 2017, 94, 416-442. [CrossRef]

38. Al-Hamdani, M. The case for stringent alcohol warning labels: Lessons from the tobacco control experience. J. Public Health Policy 2013, 35, 65-74. [CrossRef]

39. World Health Organization. Global Strategy to Reduce the Harmful Use of Alcohol; World Health Organization: Geneva, Switzerland, 2010.

40. Bates, S.; Holmes, J.; Gavens, L.; De Matos, E.G.; Li, J.; Ward, B.; Hooper, L.; Dixon, S.; Buykx, P. Awareness of alcohol as a risk factor for cancer is associated with public support for alcohol policies. BMC Public Health 2018, 18, 1-11. [CrossRef]

41. Buykx, P.; Gilligan, C.; Ward, B.; Kippen, R.; Chapman, K. Public support for alcohol policies associated with knowledge of cancer risk. Int. J. Drug Policy 2015, 26, 371-379. [CrossRef]

42. Alcohol Action Ireland What is Public Health Alcohol Act? Available online: https://alcoholireland.ie/what-is-the-publichealth-alcohol-bill/ (accessed on 11 June 2021).

43. Hassan, L.M.; Shiu, E. A systematic review of the efficacy of alcohol warning labels: Insights from qualitative and quantitative research in the new millennium. J. Soc. Mark. 2018, 8, 333-352. [CrossRef]

44. Clarke, N.; Pechey, E.; Kosīte, D.; König, L.M.; Mantzari, E.; Blackwell, A.K.M.; Marteau, T.M.; Hollands, G.J. Impact of health warning labels on selection and consumption of food and alcohol products: Systematic review with meta-analysis. Health Psychol. Rev. 2020, 1-24. [CrossRef] [PubMed]

45. Dimova, E.D.; Mitchell, D. Rapid literature review on the impact of health messaging and product information on alcohol labelling. Drugs: Educ. Prev. Policy 2021, 1-13. [CrossRef]

46. Wettlaufer, A. Can a Label Help me Drink in Moderation? A Review of the Evidence on Standard Drink Labelling. Subst. Use Misuse 2018, 53, 585-595. [CrossRef] [PubMed]

47. Arksey, H.; O’Malley, L. Scoping studies: Towards a methodological framework. Int. J. Soc. Res. Methodol. 2005, 8, 19-32. [CrossRef]

48. Tricco, A.C.; Lillie, E.; Zarin, W.; O’Brien, K.; Colquhoun, H.; Kastner, M.; Levac, D.; Ng, C.; Sharpe, J.P.; Wilson, K.; et al. A scoping review on the conduct and reporting of scoping reviews. BMC Med Res. Methodol. 2016, 16, 1-10. [CrossRef]

49. Tricco, A.C.; Lillie, E.; Zarin, W.; O’Brien, K.K.; Colquhoun, H.; Levac, D.; Moher, D.; Peters, M.D.J.; Horsley, T.; Weeks, L.; et al. PRISMA extension for scoping reviews (PRISMA-ScR): Checklist and explanation. Ann. Intern. Med. 2018, 169, 467-473. [CrossRef]

50. Pham, C.; Rundle-Thiele, S.; Parkinson, J.; Li, S. Alcohol Warning Label Awareness and Attention: A Multi-method Study. Alcohol Alcohol. 2017, 53, 39-45. [CrossRef]

51. Hobin, E.; Schoueri-Mychasiw, N.; Weerasinghe, A.; Vallance, K.; Hammond, D.; Greenfield, T.K.; McGavock, J.; Paradis, C.; Stockwell, T. Effects of strengthening alcohol labels on attention, message processing, and perceived effectiveness: A quasiexperimental study in Yukon, Canada. Int. J. Drug Policy 2020, 77, 102666. [CrossRef]

52. Hobin, E.; Weerasinghe, A.; Vallance, K.; Hammond, D.; McGavock, J.; Greenfield, T.K.; Schoueri-Mychasiw, N.; Paradis, C.; Stockwell, T. Testing Alcohol Labels as a Tool to Communicate Cancer Risk to Drinkers: A Real-World Quasi-Experimental Study. J. Stud. Alcohol Drugs 2020, 81, 249-261. [CrossRef] 
53. Zhao, J.; Stockwell, T.; Vallance, K.; Hobin, E. The Effects of Alcohol Warning Labels on Population Alcohol Consumption: An Interrupted Time Series Analysis of Alcohol Sales in Yukon, Canada. J. Stud. Alcohol Drugs 2020, 81, 225-237. [CrossRef] [PubMed]

54. Hobin, E.; Shokar, S.; Vallance, K.; Hammond, D.; McGavock, J.; Greenfield, T.K.; Schoueri-Mychasiw, N.; Paradis, C.; Stockwell, T. Communicating risks to drinkers: Testing alcohol labels with a cancer warning and national drinking guidelines in Canada. Can. J. Public Health 2020, 111, 716-725. [CrossRef] [PubMed]

55. Clarke, N.; Pechey, E.; Mantzari, E.; Blackwell, A.K.M.; De Loyde, K.; Morris, R.W.; Munafò, M.R.; Marteau, T.M.; Hollands, G.J. Impact of health warning labels communicating the risk of cancer on alcohol selection: An online experimental study. Addiction 2021, 116, 41-52. [CrossRef]

56. Clarke, N.; Blackwell, A.K.M.; De Loyde, K.; Pechey, E.; Hobson, A.; Pilling, M.; Morris, R.W.; Marteau, T.M.; Hollands, G.J. Health warning labels and alcohol selection: A randomised controlled experiment in a naturalistic shopping laboratory. Addiction 2021, 3. [CrossRef]

57. Pechey, E.; Clarke, N.; Mantzari, E.; Blackwell, A.K.M.; Deloyde, K.; Morris, R.W.; Marteau, T.M.; Hollands, G.J. Image-and-text health warning labels on alcohol and food: Potential effectiveness and acceptability. BMC Public Health 2020, 20, 1-14. [CrossRef]

58. Jongenelis, M.; Pettigrew, S.; Wakefield, M.; Slevin, T.; Pratt, I.S.; Chikritzhs, T.; Liang, W. Investigating Single-Versus MultipleSource Approaches to Communicating Health Messages Via an Online Simulation. Am. J. Health Promot. 2018, 32, 979-988. [CrossRef] [PubMed]

59. Jongenelis, M.I.; Pratt, I.S.; Slevin, T.; Chikritzhs, T.; Liang, W.; Pettigrew, S. The effect of chronic disease warning statements on alcohol-related health beliefs and consumption intentions among at-risk drinkers. Health Educ. Res. 2018, 33, 351-360. [CrossRef] [PubMed]

60. Pettigrew, S.; Jongenelis, M.I.; Glance, D.; Chikritzhs, T.; Pratt, I.S.; Slevin, T.; Liang, W.; Wakefield, M. The effect of cancer warning statements on alcohol consumption intentions. Health Educ. Res. 2016, 31, 60-69. [CrossRef]

61. Wigg, S.; Stafford, L.D. Health Warnings on Alcoholic Beverages: Perceptions of the Health Risks and Intentions towards Alcohol Consumption. PLoS ONE 2016, 11, e0153027. [CrossRef]

62. Stafford, L.D.; Salmon, J. Alcohol health warnings can influence the speed of consumption. J. Public Health 2017, 25, 147-154. [CrossRef] [PubMed]

63. Gold, N.; Egan, M.; Londakova, K.; Mottershaw, A.; Harper, H.; Burton, R.; Henn, C.; Smolar, M.; Walmsley, M.; Arambepola, R.; et al. Effect of alcohol label designs with different pictorial representations of alcohol content and health warnings on knowledge and understanding of low-risk drinking guidelines: A randomized controlled trial. Addiction 2021, 116, 1443-1459. [CrossRef]

64. Al-Hamdani, M.; Smith, S. Alcohol warning label perceptions: Emerging evidence for alcohol policy. Can. J. Public Health 2015, 106, e395-e400. [CrossRef]

65. Hall, M.G.; Grummon, A.H.; Lazard, A.J.; Maynard, O.M.; Taillie, L.S. Reactions to graphic and text health warnings for cigarettes, sugar-sweetened beverages, and alcohol: An online randomized experiment of US adults. Prev. Med. 2020, 137, 106120. [CrossRef] [PubMed]

66. Monk, R.L.; Westwood, J.; Heim, D.; Qureshi, A.W. The effect of pictorial content on attention levels and alcohol-related beliefs: An eye-tracking study. J. Appl. Soc. Psychol. 2017, 47, 158-164. [CrossRef]

67. Morgenstern, M.; Dumbili, E.W.; Hansen, J.; Hanewinkel, R. Effects of alcohol warning labels on alcohol-related cognitions among German adolescents: A factorial experiment. Addict. Behav. 2021, 117, 106868. [CrossRef] [PubMed]

68. Krischler, M.; Glock, S. Alcohol warning labels formulated as questions change alcohol-related outcome expectancies: A pilot study. Addict. Res. Theory 2015, 23, 343-349. [CrossRef]

69. Ma, Z. The Role of Narrative Pictorial Warning Labels in Communicating Alcohol-Related Cancer Risks. Health Commun. 2021, 1-9. [CrossRef]

70. Annunziata, A.; Agnoli, L.; Vecchio, R.; Charters, S.; Mariani, A. Health warnings on wine labels: A discrete choice analysis of Italian and French Generation Y consumers. Wine Econ. Policy 2019, 8, 81-90. [CrossRef]

71. Blackwell, A.K.M.; Drax, K.; Attwood, A.S.; Munafò, M.R.; Maynard, O.M. Informing drinkers: Can current UK alcohol labels be improved? Drug Alcohol Depend. 2018, 192, 163-170. [CrossRef]

72. Glock, S.; Krolak-Schwerdt, S. Changing Outcome Expectancies, Drinking Intentions, and Implicit Attitudes toward Alcohol: A Comparison of Positive Expectancy-Related and Health-Related Alcohol Warning Labels. Appl. Psychol. Health Well-Being 2013, 5, 332-347. [CrossRef]

73. Hall, M.G.; Grummon, A.H.; Maynard, O.M.; Kameny, M.R.; Jenson, D.; Popkin, B.M. Causal Language in Health Warning Labels and US Adults' Perception: A Randomized Experiment. Am. J. Public Health 2019, 109, 1429-1433. [CrossRef]

74. Jarvis, W.; Pettigrew, S. The relative influence of alcohol warning statement type on young drinkers' stated choices. Food Qual. Prefer. 2013, 28, 244-252. [CrossRef]

75. Sillero-Rejon, C.; Attwood, A.S.; Blackwell, A.K.M.; Ibáñez-Zapata, J.-A.; Munafò, M.R.; Maynard, O.M. Alcohol pictorial health warning labels: The impact of self-affirmation and health warning severity. BMC Public Health 2018, 18, 1403. [CrossRef] [PubMed]

76. Al-Hamdani, M.; Smith, S.M. Alcohol Warning Label Perceptions: Do Warning Sizes and Plain Packaging Matter? J. Stud. Alcohol Drugs 2017, 78, 79-87. [CrossRef] 
77. Jané-Llopis, E.; Kokole, D.; Neufeld, M.; Hasan, O.S.M.; Rehm, J. What Is the Current Alcohol Labelling Practice in the WHO European Region and What Are Barriers and Facilitators to Development and Implementation of Alcohol Labelling Policy? Health Evidence Network Synthesis Report, No. 68. Available online: https://www.ncbi.nlm.nih.gov/books/NBK558550/ (accessed on 11 June 2021).

78. Neufeld, M.; Ferreira-Borges, C.; Rehm, J. Implementing Health Warnings on Alcoholic Beverages: On the Leading Role of Countries of the Commonwealth of Independent States. Int. J. Environ. Res. Public Health 2020, 17, 8205. [CrossRef] [PubMed]

79. World Health Organisation. Global Status Report on Alcohol and Health 2018; World Health Organization: Geneva, Switzerland, 2018; Volume 65. Available online: http:/ / www.who.int/iris/handle/10665/112736 (accessed on 27 August 2021).

80. Chisholm, D.; Moro, D.; Bertram, M.; Pretorius, C.; Gmel, G.; Shield, K.; Rehm, J. Are the "Best Buys" for Alcohol Control Still Valid? An Update on the Comparative Cost-Effectiveness of Alcohol Control Strategies at the Global Level. J. Stud. Alcohol Drugs 2018, 79, 514-522. [CrossRef] [PubMed]

81. WHO. Status Report on Alcohol Consumption, Harm and Policy Responses in 30 European Countries 2019; WHO: Copenhagen, Denmark, 2019.

82. Moore, G.F.; Audrey, S.; Barker, M.; Bond, L.; Bonell, C.; Hardeman, W.; Moore, L.; O'Cathain, A.; Tinati, T.; Wight, D.; et al. Process evaluation of complex interventions: Medical Research Council guidance. BMJ 2015, 350. [CrossRef]

83. Stockwell, T.; Solomon, R.; O'Brien, P.; Vallance, K.; Hobin, E. Cancer Warning Labels on Alcohol Containers: A Consumer's Right to Know, a Government's Responsibility to Inform, and an Industry's Power to Thwart. J. Stud. Alcohol Drugs 2020, 81, 284-292. [CrossRef] [PubMed]

84. Petticrew, M.; Shemilt, I.; Lorenc, T.M.; Marteau, T.; Melendez-Torres, G.J.; O’Mara-Eves, A.; Stautz, K.; Thomas, J. Alcohol advertising and public health: Systems perspectives versus narrow perspectives. J. Epidemiol. Community Health 2017, 71, 308-312. [CrossRef] [PubMed]

85. O'Brien, P.; Mitchell, A.D. On the Bottle: Health Information, Alcohol Labelling and the WTO Technical Barriers to Trade Agreement. QUT Law Rev. 2018, 18, 124. [CrossRef]

86. Vallance, K.; Stockwell, T.; Hammond, D.; Shokar, S.; Schoueri-Mychasiw, N.; Greenfield, T.; McGavock, J.; Zhao, J.; Weerasinghe, A.; Hobin, E. Testing the Effectiveness of Enhanced Alcohol Warning Labels and Modifications Resulting From Alcohol Industry Interference in Yukon, Canada: Protocol for a Quasi-Experimental Study. JMIR Res. Protoc. 2020, 9, e16320. [CrossRef] [PubMed]

87. Weerasinghe, A.; Schoueri-Mychasiw, N.; Vallance, K.; Stockwell, T.; Hammond, D.; McGavock, J.; Greenfield, T.K.; Paradis, C.; Hobin, E. Improving Knowledge that Alcohol Can Cause Cancer is Associated with Consumer Support for Alcohol Policies: Findings from a Real-World Alcohol Labelling Study. Int. J. Environ. Res. Public Health 2020, 17, 398. [CrossRef] [PubMed]

88. Hiilamo, H.; Crosbie, E.; A Glantz, S. The evolution of health warning labels on cigarette packs: The role of precedents, and tobacco industry strategies to block diffusion. Tob. Control 2014, 23, e2. [CrossRef] [PubMed]

89. Myers, M.L. The FCTC's evidence-based policies remain a key to ending the tobacco epidemic. Tob. Control 2013, 22, i45-i46. [CrossRef] 